\title{
Benefits of an immunogenic personalized neoantigen nanovaccine in patients with high-risk gastric/gastroesophageal junction cancer
}

\section{Baorui Liu ( $\sim$ baoruiliu@nju.edu.cn )}

The Comprehensive Cancer Centre of Nanjing Drum Tower Hospital, The Affiliated Hospital of Nanjing University Medical School https://orcid.org/0000-0002-2539-7732

Qin Liu

The Comprehensive Cancer Centre of Nanjing Drum Tower Hospital, The Affiliated Hospital of Nanjing University Medical School

\section{Yanhong Chu}

The Comprehensive Cancer Centre of Nanjing Drum Tower Hospital, The Affiliated Hospital of Nanjing University Medical School

\section{Jie Shao}

The Comprehensive Cancer Centre of Nanjing Drum Tower Hospital, The Affiliated Hospital of Nanjing University Medical School

\section{Hanqing Qian}

The Comprehensive Cancer Centre of Nanjing Drum Tower Hospital, The Affiliated Hospital of Nanjing University Medical School

\section{Ju Yang}

The Comprehensive Cancer Centre of Nanjing Drum Tower Hospital, The Affiliated Hospital of Nanjing University Medical School

\section{Huizi Sha}

The Comprehensive Cancer Centre of Nanjing Drum Tower Hospital, The Affiliated Hospital of Nanjing University Medical School

\section{lanqi Cen}

The Comprehensive Cancer Centre of Nanjing Drum Tower Hospital, The Affiliated Hospital of Nanjing University Medical School

\section{Manman Tian}

The Comprehensive Cancer Centre of Nanjing Drum Tower Hospital, The Affiliated Hospital of Nanjing University Medical School

\section{Qiuping Xu}

The Comprehensive Cancer Centre of Nanjing Drum Tower Hospital, The Affiliated Hospital of Nanjing University Medical School

\section{Fangjun Chen}


The Comprehensive Cancer Centre of Nanjing Drum Tower Hospital, The Affiliated Hospital of Nanjing University Medical School

\section{Yang Yang}

The Comprehensive Cancer Centre of Nanjing Drum Tower Hospital, The Affiliated Hospital of Nanjing University Medical School

\section{Weifeng Wang}

OrigiMed, Shanghai, China

\section{Kai Wang}

OrigiMed, Shanghai, China

\section{Lixia Yu}

Drum-Tower Hospital, Medical School of Nanjing University

\section{Jia Wei}

The Comprehensive Cancer Centre of Nanjing Drum Tower Hospital, The Affiliated Hospital of Nanjing University Medical School

\section{Article}

Keywords: personalized neoantigen nanovaccine (PNVAC), immune response, gastric cancer recurrence

Posted Date: November 5th, 2021

DOI: https://doi.org/10.21203/rs.3.rs-952430/v1

License: (c) (i) This work is licensed under a Creative Commons Attribution 4.0 International License. Read Full License 


\section{Benefits of an immunogenic personalized neoantigen nanovaccine in patients with high-risk gastric/gastroesophageal junction cancer}

Qin Liu ${ }^{1,3}$, Yanhong $\mathrm{Chu}^{1,3}$, Jie Shao ${ }^{1}$, Hanqing Qian ${ }^{1}, \mathrm{Ju}_{\text {Yang }}{ }^{1}$, Huizi Sha ${ }^{1}$, Lanqi Cen ${ }^{1}$, Manman Tian ${ }^{1}$, Qiuping Xu ${ }^{1}$, Fanjun Chen ${ }^{1}$, Yang Yang ${ }^{1}$, Weifeng Wang ${ }^{2}$, Kai Wang $^{2}$, Lixia $\mathrm{Yu}^{1}$, Jia Wei $^{1 *}$, Baorui Liu $^{1 *}$

${ }^{1}$ The Comprehensive Cancer Centre of Drum Tower Hospital, Medical School of Nanjing University \& Clinical Cancer Institute of Nanjing University, Nanjing, China

${ }^{2}$ OrigiMed, Shanghai, China

${ }^{3}$ These authors contributed equally to this work.

* Address correspondence to:

Baorui Liu, MD, Ph.D

E-mail: baoruiliu@nju.edu.cn

Jia Wei, MD,Ph.D

E-mail: jiawei99@nju.edu.cn

The Comprehensive Cancer Centre of Drum Tower Hospital

Medical School of Nanjing University

Clinical Cancer Institute of Nanjing University

321 Zhongshan Road

Nanjing 210008 China 


\section{Abstract}

Personalized neoantigen vaccines have shown strong immunogenicity in clinical trial, but still face various challenges in facilitating an efficient antitumour immune response. Here we prepared a personalized neoantigen nanovaccine (PNVAC) platform for adjuvant cancer immunotherapy. PNVAC triggered superior protective efficacy against tumor recurrence and promoted longer survival than free neoantigens, especially when combined with anti-PD-1 treatment in a murine tumor model. A phase I clinical trial (ChiCTR1800017319) was initiated to study the safety, immunogenicity, and prophylactic effect of PNVAC on preventing tumor recurrence in patients with high-risk gastric/gastroesophageal junction cancer after adjuvant chemotherapy post-surgical resection. The 1- and 2-year disease-free survival rates were significantly higher than historical control. PNVAC induced both $\mathrm{CD}^{+}$and $\mathrm{CD}^{+} \mathrm{T}$ cell responses as well as antigen-experienced memory $\mathrm{T}$ cell phenotype. Furthermore, the immune response was persistent and remained evident one year after the vaccination. Our work provides a safe and feasible strategy for development of neoantigen vaccines to delay gastric cancer recurrence after surgery.

Neoantigens encoded by tumor-specific mutations are key targets of efficient $\mathrm{T}$ cell-mediated immunity and antitumor immune responses ${ }^{1,2}$. Neoepitopes are highly immunogenic and recognized as excellent therapeutic cancer vaccine candidates. Recent human clinical trials for melanoma and glioblastoma revealed that peptide-based neoantigen vaccines were safe and demonstrated promising signs of 
clinical efficacy and strong immunogenicity ${ }^{3-5}$. However, peptide-based neoantigen vaccines are generally challenged by tumor heterogeneity, early enzymatic degradation, and poor antigen-presenting cells (APCs) and lymph nodes (LNs) trafficking ${ }^{6}$. Thus, there is an important unmet need for additional approaches to effectively induce systemic immune responses with neoantigen-based vaccines to achieve clinical success. Nanotechnology-based cancer vaccines have the potential ability to avoid rapid in vivo clearance of payloads delivery immunomodulators to lymphoid organs and regulate the delivery of cell-impermeable compounds to immune cells, thus improving the antigen uptake of $\mathrm{APCs}^{7-9}$.

Gastric carcinoma is the fifth most commonly diagnosed cancer and the third leading cause of cancer-related death in the world ${ }^{10,11}$. The 2-year disease-free survival (DFS) rate is approximately $60 \%$ in patients with stage III gastric cancer treated by curative surgery followed by adjuvant chemotherapy ${ }^{12}$. In the CLASSIC study, the 3 -year overall survival rate was only $33 \%$ for stage IIIB gastric cancer ${ }^{13}$. In the past few years, programmed cell death 1 (PD-1) and anti-PD-1 ligand 1 (PD-L1) blockade treatment have demonstrated substantial antitumor activity in a wide range of tumor types and have revolutionized cancer management. Only a minority of metastatic gastric cancer patients demonstrated long-term clinical benefit from immune checkpoint inhibitions (ICIs) ${ }^{14}$. A novel approach is urgently needed to improve the generally poor prognosis of patients with high-risk gastric/gastroesophageal junction (G/GEJ) cancer after adjuvant chemotherapy following surgical resection. 
In this study, we prepared a personalized neoantigen nanovaccine (PNVAC) without complicated chemical appendages. PNVAC elicited potent and durable antitumor immunity in a syngeneic murine foregastric cancer model. The safety, prophylactic effect, and immunogenicity on tumor recurrence of PNVAC in patients with stage IIIB/IIIC/IVA G/GEJ cancer were evaluated. Given the biological and functional significance of PNVAC, coupled with the poor prognosis of high-risk gastric cancer, we report here results of the first-in-human phase I clinical trial to apply PNVAC in patients with G/GEJ cancer as a complementary approach for adjuvant chemotherapy.

\section{Preparation and characterization of PNVAC}

To validate the LN-targeting capacity of our vaccine platform, neoantigens of the murine foregastric cancer cell line MFC were identified by whole-exome sequencing (WES). Individual human leukocyte antigen (HLA)-binding epitopes were selected as target peptides for the vaccine design according to the predicted major histocompatibility complex (MHC)-I H-2Kk binding affinity, and multiple neoantigen peptides were conjugated to amphiphilic lipids 1,2-distearoyl-sn-glycero-3-phosphoethanolamine- $\mathrm{N}$-[hydroxysuccinimidyl(polyethyl eneglycol)] (DSPE-PEG 2000 -NHS) to generate PNVAC. ${ }^{1} \mathrm{H}$ nuclear magnetic resonance and MALDI-TOF mass spectrometry molecular weight characterization confirmed the successful conjunction of peptides (Fig. 1a, Supplementary Fig. 1a). Dynamic light scattering analysis revealed that the particle size of PNVAC peaked at 
20-30 nM and it was slightly negatively charged (Supplementary Fig. 1b,c). The amphiphilic DSPE-PEG-peptides were self-assembled with a core of DSPE and a shell of PEG-peptides, as shown by transmission electron microscopy (Fig. 1b). The size and polydispersity index of PNVAC remained stable for up to 96 hours at either $4^{\circ} \mathrm{C}$ or $37^{\circ} \mathrm{C}$ (Supplementary Fig. 1d). PNVAC or free neoantigens labeled with Cy5 were injected subcutaneously near the tail base of 615 mice to assess the biodistribution through fluorescence using an in vivo imaging system (IVIS). The fluorescence intensity decreased after injection in both groups, but PNVAC showed a stronger fluorescence signal than the free vaccines control group at 2, 24, and $48 \mathrm{~h}$ after injection (Fig. 1c, e). The axillary and inguinal LNs were harvested at 48 hours after injection and the fluorescence intensity of PNVAC was twice that of free vaccines (Fig. 1c, d; $P<0.001$ ). The blood fluorescence intensity of mice was used to semi-quantify the peripheral concentration of PNVAC. The plasma half-life of PNVAC was determined to be 24 hours (Fig. 1g). However, no fluorescence signal was detected in the group treated with free vaccines, implying that the peptides cleared and degraded rapidly. Histological sections of the inguinal LNs indicated that PNVAC accumulated mainly in the subcapsular sinus and interfollicular areas (Fig. 1f). Beyond the altered biodistribution, we found that PNVAC spatially overlapped with dendritic cells (DCs) in the LNs (Fig. 1f).

\section{The DCs activation of PNVAC}

Adjuvants played a critical role in the initiation and magnitude of the $\mathrm{T}$ cell response after vaccination. In our study, PNVAC was emulsified with incomplete 
Freund's adjuvant Montanide ISA 51, an incomplete Freund's adjuvant formulation that was found to induce $\mathrm{T}$ cell responses in previous vaccine clinical trials ${ }^{15,16}$. To test the in vitro stimulation of DCs by PNVAC, bone marrow-derived dendritic cells (BMDCs) were stimulated with the equivalent doses of free vaccines and PNVAC plus for 48 hours. Among all nine neoantigens, five neoantigen-based nanovaccines potentially expressed the mature DC phenotypic markers CD80 and CD86 on CD11 ${ }^{+}$ DCs, compared to the free neoantigens-treated group (Fig. 1h, Supplementary Fig. 2a,b). Taking the MFC-6 neoantigen as an example, $34.3 \%$ of the CD11c $\mathrm{c}^{+}$DCs showed $\mathrm{CD}^{+} 0^{+} \mathrm{CD} 86^{+}$in the PNVAC-treated group, while only $15.1 \%$ of the $\mathrm{CD} 80^{+} \mathrm{CD} 86^{+}$for the free formulation of MFC- 6 did, indicating that PNVAC is a strong immunostimulant. Blood chemistry tests, body weights, and pathological sections of main organs revealed that PNVAC did not induce severe in vivo toxicity (Supplementary Fig. 3a-d).

\section{PNVAC elicited neoantigen-specific $T$ cells and memory $T$ cells}

To validate the prophylactic and antitumor effects of PNVAC, a murine MFC foregastric carcinoma model was established and immunized with vaccines after the growing tumor was resected with a positive margin (Fig. 2a). Magnetic resonance imaging (MRI) revealed that free neoantigen vaccines showed modest benefits against tumor recurrence (Fig. 2b) compared to the blank control group, which was consistent with previous clinical studies ${ }^{17-19}$. PNVAC substantially potentiated the protective efficacy against tumor recurrence and long survival time with approximately half of 
mice surviving at 60 days (Fig. 2c-e), much better than the therapeutic responses achieved in free neoantigens group.

We then investigated whether the PNVAC elicited in vivo DCs mature and antigen-specific T cell responses. MFC tumor-bearing mice were immunized with NS, free vaccines + Montanide ISA 51, and PNVAC + Montanide ISA 51 on days 6, 8, 10, 12, and 19. It should be noted that PNVAC efficiently delivers antigens to LNs and LN-residing DCs. The inguinal LNs were harvested and digested into single-cell suspensions for flow cytometry analysis. PNVAC was verified to promote APCs uptake and presentation neoantigens and functionally induced more mature DCs in LNs (Supplementary Fig. 4a). The splenic lymphocytes were collected one week after the last vaccination. Analysis indicated that 9.01\% CD11c + DCs exhibited $\mathrm{CD}^{+} 0^{+} \mathrm{CD} 86^{+}$in free vaccines-treated group, while $31.7 \% \mathrm{CD} 11 \mathrm{c}^{+} \mathrm{DCs}$ showed $\mathrm{CD} 80^{+} \mathrm{CD} 86^{+}$for PNVAC (Fig.3a,). The PNVAC-treated group elicited remarkable 2.5- to 4.5-fold greater frequencies of neoantigen-specific $\mathrm{CD}^{+} \mathrm{T}$ cells than free vaccines (Fig. 3b, Supplementary Fig. 4b). IFN- $\gamma$ ELISPOT (enzyme-linked immune absorbent spot) showed that peptide-specific IFN- $\gamma$ secretion by splenocytes increased significantly following nanovaccine treatment (Fig. 3c). The antitumor efficacy of the isolated splenocytes was tested using a carboxyfluorescein succinimidyl ester (CFSE)/propidium iodide (PI) labelling cytotoxicity assay. MFC cancer cells were stained with CFSE for 10 minutes at $37^{\circ} \mathrm{C}$ in the dark. The splenocytes from the mice in each group were incubated with CFSE-labelled MFC cancer cells at different effector-to-target (E:T) ratios to analyze the proportion of 
dead tumor cells $\left(\mathrm{PI}^{+} \mathrm{CFSE}^{+} / \mathrm{CFSE}^{+}\right)$. The $\mathrm{CD}^{+} \mathrm{T}$ cells in PNVAC-treated group were more effective at killing MFC cells than those of the NS-treated and free-vaccines-treated groups $(P<0.001)$. The combination of PNVAC and anti-PD-1 potentiated killing tumor cell ability better than nanovaccines alone $(P<0.0001$, Supplementary Fig. 4c).

Memory $\mathrm{T}$ cells are required for long-lasting antitumor responses when direct killing is not sufficient to fully eradicate all tumor cells ${ }^{20-22}$. PNVAC induced more effector memory $\mathrm{CD}^{+} \mathrm{T}$ cells $\left(\mathrm{CD} 3^{+} \mathrm{CD} 8^{+} \mathrm{CD} 44^{+} \mathrm{CD} 62 \mathrm{~L}^{-}\right)$than the free peptides, particularly when combined with anti-PD-1 treatment (Fig. 3e). The tumor-bearing 615 mice that achieved a complete response after PNVAC treatment were simultaneously re-challenged with MFC cells in the right lower abdomen and the murine melanoma cells B16F10 in the left lower abdomen. The B16F10 cells rapidly grew into masses after implantation, while no MFC tumor growth was observed (Fig. 3d), suggesting that PNVAC successfully induced immunological memory. To further investigate the altered tumor immune microenvironment, RNA sequencing (RNA-seq) analysis was performed on tumor tissues from the PNVAC and control groups. In total, 122 genes were upregulated while 13 genes were downregulated. Cluster analysis revealed that the differentially expressed genes were found to be mainly associated with adaptive immunity (Fig. 2f). Ten genes correlated with activated MHC class I and II antigen presentation pathways and five genes correlated with the type I IFN response upregulated were significantly upregulated (Fig. 2g, h). The expression of PD-1 on neoantigen-specific $\mathrm{CD}^{+} \mathrm{T}$ cells following PNVAC 
stimulation suggested the exhaustion of these $\mathrm{T}$ cells (Supplementary Fig. 4d). PNVAC combined with anti-PD-1 treatment thus potentiated the immunotherapeutic efficacy of PNVAC or anti-PD-1 alone, leading to $66.7 \%(4 / 6)$ complete regression of tumor and prolonged mouse survival. $100 \%(6 / 6)$ of the mice survived for 40 days after inoculation (Fig. 2c, e).

\section{Clinical outcomes and immune response of PNVAC-treated G/GEJ cancer patients}

Many patients with gastric cancer are diagnosed at an advanced stage with poor prognosis. There are no further recommendations in the National Comprehensive Cancer Network (NCCN) guidelines for gastric cancer patients undergoing D2-radical gastroectomy followed by adjuvant chemotherapy ${ }^{23,24}$. Given the biological and functional significance of PNVAC, a phase I clinical trial was initiated to study the safety, immunogenicity, and prophylactic effect on tumor recurrence of PNVAC in patients with stage IIIB/IIIC/IVA gastric cancer after surgery. The neoantigens were selected based on tumor-specific mutations identified using WES and RNA-seq. The cancer-testis antigens (CTAs) were selected according to the immunohistochemical staining of the resected tumor tissues and HLA-binding affinity prediction. PNVAC combined with adjuvant Montanide ISA 51 was administered subcutaneously to the corresponding 29 patients following completion of adjuvant chemotherapy (capecitabine/S-1+oxaliplatin or S-1+docetaxel) on days $1,4,8,15,22,43,64,85$, 
and 169 (Fig. 4a). Four injections for four cases were delayed due to COVID-19 restrictions.

Of the 30 enrolled patients, one patient withdrew due to disease progression during adjuvant chemotherapy. The patients' median age was 57 years old (range: 34-75 years), and their Eastern Cooperative Oncology Group performance status was either 0 or 1 (Supplementary Table 1). We detected an average of 186 somatic variants with a median of 153 single-nucleotide variants per tumor using WES, and RNA-seq was performed to confirm the gene expressions. A median of 183.3 HLA binders with an average half-maximum inhibitory concentration $<500 \mathrm{nM}$ were predicted per tumor. The median of detected somatic mutations was 153 (range: 75-158). Each patient received approximately 9 immunizing peptides (range: 6-13) with lengths of 9-15 amino acids (Supplementary Table 2). The median time from the last adjuvant chemotherapy cycle to vaccine administration was 5.1 weeks. In total, 29 patients received an initial vaccination and 27 patients completed the full series, comprising five priming shots and four booster shots. The repeated doses were well tolerated and no severe adverse events occurred. Twelve patients (41.4\%) developed grade 1 local skin reactions with redness, swelling, or subcutaneous indurations at the injection sites and two patients $(6.9 \%)$ developed grade 2 local skin reactions. Five patients $(17.2 \%)$ had a grade 1 or 2 fever after injection. Two patients (6.9\%) developed grade 2 myalgia (Supplementary Table 3). The median follow-up time after surgery was 26.4 months (range: 15-39.7 months). The 1- and 2-year survival rates were $96.55 \%(28 / 29)$ and $83.87 \%$ (14/17), respectively (Fig. 4b). Only one patient 
demonstrated an anastomotic recurrence at the beginning of vaccination within one year after surgery. The clinical outcome was superior to the previous study, which reported a disease-free survival of 17 months for stage IIIB and 16 months for stage IIIC gastric cancer patients treated with a D2-radical gastroectomy followed adjuvant chemotherapy ${ }^{25}$.

Significant accumulation of the nanovaccines was observed in the draining LNs are not limited to in vivo murine experiments. Similar evidence was also displayed in the clinical study. The LNs of patient 005 were observed to be swollen and draining on day 2 after the last shot, but they had returned to normal on day 490 (Fig. 4c), indirectly indicating the efficient LNs uptake of the administered PNVAC. The patients' immune responses to PNVAC were evaluated by detecting and motoring the IFN- $\gamma$ released from peripheral blood mononuclear cells (PBMCs) using various assays, including ex vivo or in vitro cytometric bead arrays (CBA), IFN- $\gamma$ ELISPOT assay and intracellular cytokine staining (ICS). The immunized antigens elicited T cell responses against all patients and $93.1 \%$ of patients generated novel immune responses. Furthermore, $76.2 \%(202 / 265)$ of immunized peptides induced positive T cells because the IFN- $\gamma$ secretion was double that of the medium (no peptide) control (Fig. 4d, e, Supplementary Fig. 5-7). When monitored by an ex vivo IFN- $\gamma$ CBA assay, approximately $23.9 \%(42 / 176)$ of the immunized neoantigens induced primary responses and 43.8\% (77/176) induced novel antigen reactive responses after repeated PNVAC treatment (Fig.4d, Supplementary Fig. 5, 6). In some cases, the reactivity of $\mathrm{T}$ cells was assessed using in vitro IFN- $\gamma$ CBA assay. The primary and novel 
responses to the given neoantigens were found to be $42.7 \%(38 / 89)$ and $42.7 \%$ (38/89), when the immune response was evaluated by in vitro stimulation (Fig.4e, Supplementary Fig. 7). Immunizing peptides 01 and 06 (Pep01 and Pep06, respectively) were used to test the immune response of patient 035 after vaccination. In vitro IFN- $\gamma$ ELISPOT showed that IFN- $\gamma$ secretion by PBMCs against Pep01 and Pep06 were undetectable before the vaccination but evident at six weeks following treatment by PNVAC (Fig. 5a). When the immunizing peptides were mixed into a peptide pool to stimulate $\mathrm{PBMCs}$, memory $\mathrm{CD}^{+} \mathrm{T}$ cells were the dominant subpopulation of the IFN- $\gamma$-producing antigen-reactive $\mathrm{CD}^{+} \mathrm{T}$ cells (Fig. $5 \mathrm{~b}$ ). In vitro ICS showed Pep01 and Pep06 elicited both $\mathrm{CD}^{+} \mathrm{T}$ cells and $\mathrm{CD} 8^{+} \mathrm{T}$ cells that expressed IFN- $\gamma$, TNF- $\alpha$ and IL-2 cytokines (Fig. 5c, d). The proportion of TNF- $\alpha$ producing $\mathrm{CD}^{+} \mathrm{T}$ cells was significantly elevated in 21 patients at 24 weeks after vaccination (Fig. 5e). CD4 $4^{+} \mathrm{IFN}-\gamma^{+}, \mathrm{CD} 8^{+} \mathrm{IFN}-\gamma^{+}$, and $\mathrm{CD} 8^{+} \mathrm{TNF}-\alpha^{+} \mathrm{T}$ cell frequencies tended to increase after vaccination, although the differences were not statistically significant (Supplementary Fig. 8a-c). In 12 patients, $52.3 \%$ of the immunized neoepitopes retained detectable $\mathrm{T}$ cell activity 1 year after vaccination (Fig. $5 \mathrm{f}$ ). The PBMCs of patient 056 , showed stronger reactivity against 5 out of 7 immunizing peptides at approximately 51 weeks after PNVAC treatment (Fig. 5g). ICS also demonstrated that the development of a sustained immune response following PNVAC vaccination (Fig. 5h). Taking patient 002 as an example, more than $20 \%$ of the neoantigen-reactive $\mathrm{CD}^{+}$and $\mathrm{CD}^{+} \mathrm{T}$ cells were polyfunctional at 18 weeks post-vaccination, as detected by evaluating the secretion of one or two inflammatory 
cytokines (Fig. 5i, Supplementary Fig. 9). In addition to exhibiting effectiveness, the immune responses induced by PNVAC were persistent, as indicated by the increasement of polyfunctional $\mathrm{CD}^{+}$and $\mathrm{CD}^{+} \mathrm{T}$ cells observed at 110 weeks post-vaccination (Fig. 5i, Supplementary Fig. 9).

Patient 023, a 67-year-old man with PD-L1 negative stage IIIC gastric carcinoma, received PNVAC after 6 cycles of adjuvant chemotherapy. Four weeks after the first vaccination, he developed grade 2 local skin reactions with subcutaneous indurations. A positron emission tomography-computed tomography (PET/CT) scan revealed concurrently increased FDG uptake in subcutaneous indurations and residual stomach (Fig. 6a), indicating the active inflammation of injection sites. Approximately 46 weeks after vaccination, the disease progressed with retroperitoneal lymphadenopathy (Fig. 6c). After anti-PD-1 antibody monotherapy, inconsistent responses were observed, with apparent shrinking of the swollen LNs and new-onset ascites as well as anastomosis relapse (Fig. 6c). Immune responses to each immunizing peptide were observed at the beginning of relapse (week 46) and were noticeably enhanced to 5 out of the 8 immunizing peptides after treatment with an anti-PD-1 antibody (Fig. 6a). A previous study of personal neoantigen vaccination reported that 2 out of 6 melanoma patients who developed recurrent disease in the trial experienced expansion of neoantigen-specific $\mathrm{T}$ cells and achieved complete regression after anti-PD-1 treatment ${ }^{5}$. An enhanced immune response was also detected in patient 023 following PD-1 blockade, suggesting the synergistic effects of neoantigen vaccine and anti-PD-1 therapy, which is consistent with the preclinical findings. Re-biopsy was 
performed under endoscopy after the development of anastomotic obstruction. Interestingly, the progressed lesion sample after anti-PD-1 therapy was detected entirely different gene mutations compared to the previous surgical sample (Fig. 6d) and none of the mutations corresponding to the patient's immunized neoantigens were detected in the progressed lesion (Fig. 6e).

\section{Conclusions}

Conventional tumor therapeutic vaccines usually select tumor-associated proteins that are highly expressed by tumor cells as targets, but showed limited therapeutic efficacy because the proteins are also expressed by nonmalignant cells ${ }^{16,26,27}$. Neoepitopes derived from somatic mutation can be processed and presented on MHC molecules and have a high affinity to $\mathrm{T}$ cell receptors ${ }^{28,29}$. CTAs, which are highly expressed in the tumors and testis but not expressed in normal tissues, are also favorable cancer vaccine targets ${ }^{30,31}$. Insufficient trafficking to lymphoid organs as well as delivery antigens to immune cells are responsible to limited clinical efficacy ${ }^{32,33}$. The number of tumor-infiltrating $T$ cells in the PNVAC-treated group was significantly higher than that of the free vaccine-treated group, suggesting that the neoantigen-specific $\mathrm{T}$ cells generated by PNVAC are capable of trafficking to tumor sites. The IFN- $\gamma$-producing neoantigen reactive $\mathrm{T}$ cells highly expressed memory T-cell phenotypic markers, indicating that patients with prior PNVAC treatment developed durable long-term anti-tumor control. As a result, the administration of nanovaccines triggered a more significant tumor shrinking, and a 
longer survival time than traditional peptide-based vaccines. Adjuvants are conventionally used to improve immunogenicity clinically ${ }^{34}$. However, the discrepancy between antigen peptides and adjuvants with regard to their pharmacokinetics and physicochemical properties is a major obstacle in the development of therapeutic cancer vaccines ${ }^{35,36}$. For a vaccine adjuvant, we employed topically applied Montanide ISA 51. By strategically loading multiple neoantigens and adjuvants in the same nanoparticle, the PNVAC mediated efficient antigen and adjuvant codelivery to LNs and APCs. In the PNVAC-treated group, we found that genes related to antigen processing and type I IFN signaling were upregulated, suggesting that PNVAC promoted antigen presentation and process and potent $\mathrm{T}$ cell cytotoxicity to targeted tumor cells. The mice whose tumors were eradicated by PNVAC were still tumor-free after re-challenge because PNVAC augmented neoantigen-specific and effector memory $\mathrm{T}$ cell responses.

Resected stage IIIB-IVA gastric cancer is highly incurable, with high risks of recurrence and metastasis. In addition to the current strategies of definitive surgery and adjuvant chemotherapy, a novel strategy is needed to improve the poor prognosis of gastric cancer. For the first time, we integrated the personalized neoantigens/CTAs with this novel vaccine platform to generate PNVAC. The PNVAC platform not only showed an improved LN-targeting capacity and a significant $\mathrm{CD}^{+}$and $\mathrm{CD} 8^{+} \mathrm{T}$ cells' mediated immune response in the preclinical studies, but also clearly prolonged DFS in patients with high-risk G/GEJ cancer. $\mathrm{T}$ cell responses against $65 \%$ immunized neoepitopes were detected in the enrolled 29 patients, indicating certain epitope 
prediction accuracy in a large cohort of patients and the possibility that a certain frequency epitope-specific T cells were present before vaccination. Epitope spreading in the previous study was associated with prolonged progression-free survival ${ }^{37}$. In our study, most patients developed obvious epitope spreading because neoantigen-directed responses were enhanced after PNVAC treatment.

Overall, we demonstrated that a personalized neoantigen-based nanovaccine is safe and feasible for combatting gastric carcinomas, which typically are relatively unresponsive to PD-1 inhibitors ${ }^{38-40}$. A larger randomized clinical trial is needed to confirm and validate the possibility of neoantigen nanovaccine design for G/GEJ cancer patients in future.

\section{Methods}

Tumor cell line. MFC murine foregastric carcinoma cell line (Shanghai Institute for Biological Science, China) was cultured in RPMI 1640 medium with $10 \%$ fetal calf serum (FCS) at $37^{\circ} \mathrm{C}$ under an atmosphere of $5 \% \mathrm{CO}_{2}$. Cells were sub-cultured at $80 \%$ confluence approximately every 2 to 3 days at a split ratio of $1: 3$. Female 615 mice (6-8 weeks old) were purchased from Shanghai Super-B\&K Laboratory Animal Corp. Ltd. (Shanghai, China) and all experiments were conducted in accordance with the relevant guidelines and regulations for animal research at the Affiliated Nanjing Drum Tower Hospital of Nanjing University Medical School.

Neoantigen prediction. Tumor tissue specimens and blood samples were collected from MFC tumor-bearing mice and WES was performed (Shanghai Biotecan 
Pharmaceuticals Co., Ltd, Shanghai, China). The nonsynonymous mutations identified were sorted according to frequency and the PubMed nucleotide tool was used to identify amino acids of wild-type proteins. The binding affinity of mutant peptides to $\mathrm{HLA} \mathrm{H}_{2}-\mathrm{K}^{\mathrm{K}}$ was determined using netMHCpan v4.0. 9-mer and 10-mer mutant peptides with a binding affinity half-maximum inhibitory concentration $<500 \mathrm{nM}$ or percentage rank $<2.0$ were selected and synthesized as cancer vaccines. Nine mutant epitopes for MFC cells, including MFC-1 (NEIVMFTLI), MFC-2 (MELLGHGLV), MFC-3 (VENVAWAHI), MFC-4 (LEMSFYWSL), MFC-5 (IEFIRKFAV), MFC-6 (LEMSFYWSLV), MFC-7 (MELACSSTYV), MFC-8 (YVENVAWAHI), and MFC-9 (INEIVMFTLI), were synthesized.

Preparation and characterization of PNVAC nanovaccines. The general procedure for PNVAC preparation is described below. 2-Distearoyl-sn-glycero-3-phosphoethanolamine-N-[hydroxysuccinimidyl (polyethylene glycol)-2000] (DSPE-PEG $2000-\mathrm{NHS}$ ) and individual peptides at a molar ratio of 1:1.5 were mixed in PBS. The mixture was agitated overnight with a magnetic stirrer at room temperature. Finally, the amphiphilic polymers were purified through dialysis, lyophilized, and stored at $-80^{\circ} \mathrm{C}$ for further use. PNVAC size and zeta potential were measured in triplicate using a dynamic light scattering analyzer (Malvern Instruments Corporation, United Kingdom). To assess its stability, samples of prepared PNVAC were pre-incubated with PBS for 120 hours at $4{ }^{\circ} \mathrm{C}$ and $37^{\circ} \mathrm{C}$. 
Electron microscopy was performed using an electron microscope equipped with a CCD camera and operating at $80 \mathrm{kemV}$.

In vivo near infrared imaging. The uptake and distribution of PNVACs were evaluated by in vivo fluorescence imaging using an optical and X-ray small animal imaging system (IVIS Lumina, Perkin Elmer, Germany). A model antigen MFC-2 was labeled with Cy5 (excitation, $650 \mathrm{nM}$; emission, $680 \mathrm{nM}$ ). The Cy5-labeled free vaccines and nanovaccines were adjusted to equal $\mathrm{Cy} 5$ concentration based on photon count values and subcutaneously injected into separate 615 mice (20 $\mu \mathrm{g}$ peptide). The fluorescence signals were examined at 2, 24, and 48 hours. The inguinal LNs were collected at 48 hours to assess the concentration of nanoparticles (NPs) in the draining LNs $(n=3)$. The exposure time of each image was fixed, and fluorescence intensities obtained using the software was used for parallel comparisons.

Biodistribution study. For the biodistribution study, mice were randomized into 3 nanovaccine experimental groups. Different doses of peptides $(100,300$, or $900 \mu \mathrm{g})$ in Cy5-labelled nanovaccines mixed with Montanide ISA 51 (SEPPIC, France) were administered subcutaneously at the tail base of the 615 mice. Blood was collected from the orbital sinus to examine the fluorescence intensity via optical and X-ray small animal imaging systems (IVIS Lumina, Perkin Elmer, Germany) at different time points.

Immunofluorescence staining. Using FITC-labelled OVA as a model antigen, inguinal LNs were acquired at 48 hours following PNVAC injection. For colocalization studies with DCs and T cells, $8-\mu \mathrm{m}$ frozen sections of the LNs were 
stained with anti-CD11c and anti-CD3 antibodies. The sections were blocked using PBS supplemented with $10 \%$ goat serum for 1 hour at room temperature in the dark and stained with anti-CD3 antibody (Abcam, United Kingdom) and anti-CD11c rabbit monoclonal antibody (Cell Signaling Technology, United States) overnight at $4^{\circ} \mathrm{C}$. After washing with PBS 3 times, the sections were stained with goat anti-rabbit IgG H\&L (Cy3) (Abcam, United Kingdom), goat anti-rat IgG H\&L (Cy5) (Abcam, United Kingdom) and DAPI (Sangon Biotech, China). Immunofluorescence images were obtained using a confocal microscope (Leica, Germany).

Preparation of tissue and cell. Peripheral blood samples were collected from the orbital sinus. Spleens, LNs, and tumors were stored in NS. Single-cell suspensions were prepared in NS from spleens and LNs by grinding the tissue with the frosted surfaces of glass slides. Erythrocytes were removed with red blood cell lysis buffer. Tumors were cut into small pieces, digested with collagenase IV (1 $\mathrm{mg} \mathrm{mL}^{-1}$; Sigma), and passed through cell strainers. Bone marrow DCs were flushed from the femur and tibia, homogenized and filtered, followed by erythrocytes removal with red blood cell lysis buffer.

Flow cytometry. All samples tested were stained with indicated extracellular antibodies for $20 \mathrm{~min}$ at $4^{\circ} \mathrm{C}$ in the dark, and washed before analysis. To quantify neoantigen-specific $\mathrm{CD} 8^{+} \mathrm{T}$ cells 1 week after the last immunization by peptide-MHC (pMHC) multimer staining, samples were incubated with a mixture of H-2Kk ${ }^{\mathrm{k}}$ PE/MFC-1 (MFC-2 or MFC-3-MFC-9; BD Biosciences, United States) dimers for 30 minutes at $4^{\circ} \mathrm{C}$ in the dark. After washing, anti-CD3-FITC and 
anti-CD8-APC antibodies were added for an additional 20 minutes. To detect the frequency of regulatory $\mathrm{T}$ cells, the nuclear membranes of the cells in samples were disrupted and the cells were fixed for 1 hour in the dark with a True-Nuclear Transcription Factor Buffer Set (BioLegend, United States) following extracellular staining with anti-CD3-FITC, anti-CD4-PE/Cy7, and anti-CD25-APC antibodies. The samples were then stained with anti-Foxp3-PE antibody for 30 minutes at $4{ }^{\circ} \mathrm{C}$ in the dark. Flow cytometric data were acquired using a BD Accuri C6 cytometer (BD Biosciences, United States) and analyzed using FlowJo v10 software (TreeStar, United States). The BD ${ }^{\mathrm{TM}}$ CBA Mouse Th1/Th2 Cytokine Kit, IL-6 Flex Set, and IL-10 Flex Set were used to detect multiple cytokines (IL-2, IL-4, IL-5, IFN $\gamma$, TNF $\alpha$, IL-6, and IL-10).

In vitro cytotoxicity assay. Freshly isolated splenocytes were tested using a carboxyfluorescein succinimidyl ester (CFSE)/propidium iodide (PI) labelling cytotoxicity assay. MFC gastric cancer cells were stained with CFSE (Invitrogen, United States) for 10 minutes at $37^{\circ} \mathrm{C}$ in the dark. Splenocytes from mice in each group were then incubated with CFSE-labelled MFC cancer cells at E:T ratios of 12.5:1, 25:1, and $50: 1$ at $37^{\circ} \mathrm{C}$ and $5 \% \mathrm{CO}_{2}$. Six hours later, PI was added to the mixed cells, which were then incubated for 20 minutes at $4^{\circ} \mathrm{C}$ in the dark. Flow cytometry was performed to examine the proportion of dead tumor cells.

IFN- $\gamma$ ELISPOT assay. An IFN- $\gamma$ ELISPOT kit (Dakewei, China) was used to evaluate the cytokine secretion of $\mathrm{T}$ cells after overnight activation with peptides. Briefly, freshly isolated splenocytes from 615 mice in each group (NS, free vaccines, 
nanovaccines, $1 \times 10^{5}$ per well, $100 \mu \mathrm{L}$ AIM-V medium containing $10 \%$ FCS per well) were added to wells with peptides $\left(50 \mu \mathrm{g} \mathrm{ml}^{-1}\right)$ in triplicate and incubated for 18 hours at $37^{\circ} \mathrm{C}$. The cells were then lysed with cold water and the wells were washed before sequential incubations with biotinylated anti-IFN- $\gamma$ antibody and streptavidin-AP for 1 hour at $37^{\circ} \mathrm{C}$. A 3-amino-9-ethylcarbazole (AEC) solution mixture was then added and the plates were incubated in the dark for approximately 25 minutes at $37^{\circ} \mathrm{C}$. Finally, all wells were scanned and analyzed using an ELISPOT CTL Reader (Cell Technology Inc., Columbia, MD, United States).

Tumor model. Protective immunity: To establish a gastric cancer model, $2 \times 10^{6}$ MFC cells were injected subcutaneously into mice. The tumors were removed when they reached a volume of $\sim 100 \mathrm{~mm}^{3}$. The mice were randomly divided into 6 groups and injected subcutaneously with NS, blank vehicle, free vaccines, nanovaccines, anti-PD-1 antibody (5 mg/kg, injected intraperitoneally), or nanovaccines + anti-PD-1 (injected intraperitoneally). The mice were immunized 5 times with PNVAC (20 $\mu \mathrm{g}$ per peptide) on the stated days. The size of the tumors were measured in an unblinded manner with calipers every 2 days and their volume was calculated using the equation $\left(a^{2} \times b\right) / 2$ ( $a$, width; $b$, length). The mice were euthanized upon exhibiting signs of impaired health or when the tumor length exceeded $15 \mathrm{~mm}$.

Re-challenge model: Tumor-bearing mice cured by PNVAC were re-challenged with MFC tumor cells and B16F10 melanoma cells on opposite sides of the abdomen. Analysis of changes in gene expression. Total RNA was extracted from NS- or nanovaccine-treated tumors using Trizol Reagent (Qiagen, China). The concentration 
and quality of the isolated total RNA were determined by an Agilent 2100/2200 Bioanalyzer (Agilent Technologies, Palo Alto, CA, United States) and a NanoDrop (Thermo Fisher Scientific Inc.). One microgram of total RNA was used for mRNA sequencing on an Illumina HiSeq/NovaSeq or MGI2000 (Genewiz, China). Genes expressed differentially between the NS- and nanovaccine-treated tumors were detected using HTSeq (v0.6.1) and the DESeq2 Bioconductor package (fold change $\geq 1.5$ and $P<0.05)$ and plotted on a heat map. Ingenuity pathway analysis software (Qiagen, China) was used to explore the expression of the gene pathways of immunity. The associations of some differentially expressed genes with type I IFN responses and the MHC I and MHC II pathways were analyzed by Gene Ontology and Kyoto Encyclopedia of Genes and Genomes enrichment analyses. Their expressions in tumor-infiltrating DCs were summarized from RNA-seq data and plotted on a heat map.

Clinical trial design. Between 2018 and 2021, a single-arm, open-label first-in-human trial (ChiCTR1800017319) was conducted at the Cancer Centre of Nanjing Drum Tower Hospital in China and in accordance with the Declaration of Helsinki, International Conference on Harmonization/GCP guidelines. The study procedure was approved and supervised by the relevant authority and ethics committee of Nanjing Drum Tower Hospital. Patients were enrolled from July 2018 to July 2020. The enrollment dates for the first and last patient were July 26, 2018 and July 02, 2020. Data analysis was performed with data cutoff 31 Dec 2020. Patients with an Eastern Cooperative Oncology Group score of 0-1 and a life expectancy of at 
least 6 months were enrolled. Patient identification logs were kept strictly confidential by the investigators (see the Chinese Clinical Trial Registry, http://www.chictr.org.cn/showproj.aspx?proj=27889, for details on the eligibility criteria). All patients provided written informed consent before enrollment. Patient-specific neoantigens were selected on the basis of tumor-specific mutations identified by WES and RNA-seq. CTAs were tested using immunohistochemical staining and HLA-binding affinity prediction. The customized peptides encoding individual neoantigens were prepared in a pharmacy under GMP conditions.

Patients with Stage IIIB/IIIC/IVA G/GEJ cancers who completed or could not tolerate 6 cycles of adjuvant chemotherapy (capecitabine/S-1/oxaliplatin or S-1/docetaxel) were treated with PNVAC. PNVAC was designed and manufactured to be administered during adjuvant chemotherapy. PNVAC treatment started within 2 months after the last adjuvant chemotherapy cycle. Each patient received a $500 \mu \mathrm{g}$ dose per peptide of PNVAC by subcutaneous injection on days $1,4,8,15,22,43,64$, 85 and 169, along with adjuvant chemotherapy in the form of Montanide ISA 51 VG and $100 \mu \mathrm{g}$ GM-CSF. Cyclophosphamide $\left(200 \mathrm{mg} / \mathrm{m}^{2}\right)$ was administered before PNVAC injection on days $1,22,43,64,85$, and 169. PBMCs were isolated using a Ficoll density gradient centrifugation of heparinized blood samples from the patients and stored at $-80^{\circ} \mathrm{C}$ for immune response measurements on days $1,22,43,64$, and 129 before each dose of the vaccines was administered.

\section{Generation of personalized neoantigens.}


WES. Paired blood and tumor tissue were collected from patients and gDNA was extracted for library preparation. For gDNA extraction, formalin-fixed paraffin-embedded (FFPE) tumor sections and corresponding blood were processed. Then, 50-250 ng double-stranded DNA was fragmented to 250 bp using sonication, followed by library construction with a KAPA Hyper Prep Kit (KAPA Biosystems). Probes designed at OrigiMed, Shanghai, covering the exonic regions of more than 20,000 human coding genes, as well as introns with high pathogenic fusion frequency, were used to detect mutations. Sequencing was performed using an Illumina NovaSeq 6000 (Illumina Inc., United States) with a mean depth of $500 \times$ at the OrigiMed, a laboratory certified by the College of American Pathologists and Clinical Laboratory Improvement Amendments "YuanSu" panel. For patients 002, 003, 005, and 041, nonsynonymous mutations were identified using an OrigiMed custom hybridization capture panel, which encodes exons of 450 key cancer-related genes and selected introns of 36 genes commonly rearranged in solid tumors. The probe density was increased to ensure capture efficiency in the conservatively low-read depth region. Targeted library fragments were captured according to the protocol of hybridization capture of DNA libraries using xGen Lockdown Probes and Reagents (Integrated DNA Technologies, San Diego, CA, United States). Post-capture libraries were sequenced with a mean coverage of $900 \times$ for FFPE samples and $300 \times$ for matched blood samples on an Illumina NovaSeq 6000 platform (Illumina Inc., United States).

RNA-seq. RNA was extracted from unstained FFPE sections using the miRNeasy FFPE Kit (cat\# 217504, Qiagen) according to the manufacturer's protocol. 
The yield and quality of extracted RNA were assessed using the Qubit ${ }^{\mathrm{TM}}$ RNA HS Assay Kit (ThermoFisher Scientific) and LabChip GX Touch HT Nucleic Acid Analyzer (Perkin Elmer), respectively. After ribosomal RNA depletion by the NEBNex rRNA Depletion Kit (cat\# E6310L, New England Biolabs), cDNA synthesis was performed using M-MLV RT RNase (H-) (cat\# M3683, Promega) and an NEB Second Strand mRNA synthesis kit (cat\#E6111L, New England Biolabs). Sample library preparation was performed using a KAPA Hyper Prep Kit (KAPA Biosystems) and sequenced on a NovaSeq 6000 platform with $2 \times 151$ bp paired-end reads according to the manufacturer's instructions. The relative abundance of each annotated transcript was reported as transcripts-per-million and log2-transformed before analysis. Sequencing was performed and analyzed by OrigiMed. The data that support the findings of this study have been deposited into CNGB Sequence Archive $(\mathrm{CNSA})^{41}$ of China National GeneBank DataBase $(\mathrm{CNGBdb})^{42}$ with accession number CNP0002214.

Prediction and identification of target epitopes. OptiType was used to identify class I HLA (HLA-A, HLA-B, and HLA-C) alleles and class II HLA-DRB1 alleles. After all nonsynonymous mutations were identified using next generation sequencing (NGS), the mutant epitopes were predicted using NetMHCpan v3.1 and NetMHCIIpan v1.2 tools as described previously ${ }^{2}$. Substrings within the 15 mers that had a binding affinity of less than $500 \mathrm{nM}$ or $\%$ rank $<2.0$ for any patient's HLA allele were considered to be candidates and synthesized. 
Ex vivo and in vitro evaluation of the neoantigens' immunogenicity. Briefly, frozen PBMCs were thawed and cultured in culture AIM-V medium supplement with $10 \%$ FCS. For ex vivo analysis with the IFN- $\gamma$ Flex Set, $2 \times 10^{5}$ PBMCs were plated in 96-well cell culture plates with individual peptides $(25 \mu \mathrm{g} \mathrm{mL}-1)$ and incubated overnight; the supernatants were then harvested for further testing. The no-peptide media and stimulus phytohemagglutinin were used as negative and positive controls, respectively. For in vitro stimulation of antigen-specific T cells, $25 \mathrm{ng} \mathrm{mL}^{-1} \mathrm{IL}-7$ (PeproTech, United States) was added to the culture medium and $20 \mathrm{U} \mathrm{ml}^{-1} \mathrm{IL}-2$ (PeproTech, United States) was added on day 3. Half of the medium with additional cytokines was replaced every 3 days. After 1 day of stimulation, cells were harvested for further immune response analysis.

Cytometric Bead Array analysis of cytokines. The concentrations of IFN- $\gamma$ in culture supernatants for ex vivo and in vitro stimulation study were measured using CBA according to the manufacturer's protocol (BD Biosciences, United States). The samples were subjected to a CytoFLEX LX flow cytometer (Beckman Coulter, United States) and the data were analyzed. The IFN- $\gamma$ of mutant peptide-stimulated PBMCs being more than double that of no-peptide control was identified as indicating positive PBMCs reactivity.

IFN- $\gamma$ ELISPOT assay. For in vitro pre-stimulated PBMCs, the secretion of IFN- $\gamma$ released from $\mathrm{T}$ cells was evaluated using an IFN- $\gamma$ ELISPOT kit (Dakewei, China) after overnight stimulation with irradiated autogenous PBMCs loaded with a corresponding peptide. ${ }^{2}$ Briefly, pre-stimulated PBMCs $\left(10^{5}\right.$ per well $)$ with irradiated 
autogenous PBMC loaded with a corresponding peptide $\left(25 \mu \mathrm{g} \mathrm{mL}^{-1}\right)$ were added to wells in triplicate for 18-20 hours of incubation in AIM-V culture medium. After washing, the diluted detection antibody was added for 1 hour of incubation at $37^{\circ} \mathrm{C}$. Streptavidin-HRP (1:100 dilution) and 3-Amino-9-ethylcarbazole solution mixture were then added to each well in turn. After the plates were kept in the dark for approximately 20 minutes at room temperature, deionized water was added to stop the reaction. The plates were scanned under an ELISPOT CTL Reader (Cellular Technology Inc.) and the data were analyzed using ELISPOT software (AID). The positive PBMC reactivity was scored when spots were more than twice the size of the negative control.

Intracellular cytokine staining. In vitro multiparameter ICS assays were performed as previously described. ${ }^{5}$ Briefly, frozen PBMCs were thawed and cultured in media supplement with cytokines for 11 days of stimulation, ICS was then performed. A total of $1 \times 10^{6}$ antigenic peptide-stimulated PBMCs per well were re-stimulated with irradiated autogenous PBMC at an E:T of 2:1, and pooled peptides (each at 1.5-2.0 $\mu \mathrm{g} \mathrm{ml}^{-1}$ ) were added to the culture. The $\mathrm{T}$ cells were treated with GolgiStop (BD Biosciences) according to the manufacturer's protocols for 8 hours the following day. The stimulated $\mathrm{T}$ cells were then stained for 30 minutes at room temperature with a fixable live/dead stain (FVS780), anti-CD3 (BUV395), anti-CD4 (FITC), anti-CD8

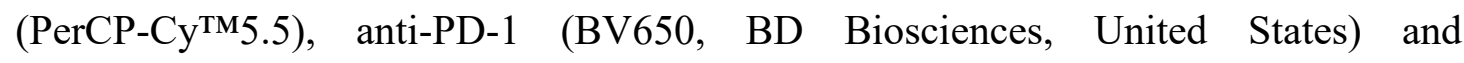
anti-CD45RO (PE-Cy' ${ }^{\mathrm{TM}}$ ) antibodies (BioLegend, United States). A Fixation/Permeabilization Solution Kit was used to fix and permeabilize the cells (BD 
Biosciences, United States). After that, anti-IFN- $\gamma$ (PE), anti-IL-2 (APC-R700) and anti-TNF- $\alpha$ (APC) antibodies (BD Biosciences) were added to stain the cells in order to measure intracellular cytokines at $4{ }^{\circ} \mathrm{C}$. The cells were washed with permeabilization buffer and fixed with $1 \%$ paraformaldehyde solution (Sigma-Aldrich). Flow cytometry was performed and the data were analyzed using FlowJo v10 software. Live and dead lymphocytes were stained and $\mathrm{CD}^{+} \mathrm{T}$ cells were gated. $\mathrm{CD}^{+}$and $\mathrm{CD} 8^{+} \mathrm{T}$ cells were separately gated and cytokine staining was plotted for each population (Supplementary Fig. 10).

Statistical analyses. For all experiments, biological replicates were performed unless otherwise stated. Comparisons between two groups were analyzed using paired two-tailed Student's $t$-test. One-way analysis of variance (ANOVA) or two-way ANOVA was performed to compare several groups. Survival benefit was determined with the log-rank test. $* P \leq 0.05, * * P \leq 0.01$, and $* * * P \leq 0.001$ were considered statistically significant.

\section{Acknowledgments}

This work has been supported by the National Natural Science Foundation of China (No. 81930080, 81972309) and the fundings for Clinical Trials from the Affiliated Drum Tower Hospital, Medical School of Nanjing University. 


\section{Author Contributions}

Baorui Liu was the principal investigator of this trial. Baorui Liu and Qin Liu designed the overall study, performed experimental and data analysis with Jia Wei, Yanhong Chu, Jie Shao and Manman Tian. Baorui Liu, Qin Liu, Jia Wei, and Yang Yang provided patient for enrollment; Yanhong Chu, Jie Shao, Hanqing Qian, Lanqi Cen Manman Tian and Lixia Yu performed experiments and data analyses. Weifeng Wang and Kai Wang performed the WES and RNA-Seq. Qin Liu, Yanhong Chu, Fangjun Chen and Weifeng Wang analysed sequencing data, selected neoantigens, and prepared nanovaccines. Qin Liu, Huizi Sha and Qiuping Xu collected the samples and coordinated clinical trial. Baorui Liu, Qin Liu, Jia Wei, Jie Shao and Ju Yang wrote the manuscript, discussed and interpreted results.

\section{Competing interests}

All authors declare that there are no conflicts of interest. 


\section{References}

1. Schumacher, T.N. \& Schreiber, R.D. Neoantigens in cancer immunotherapy. Science 348,

69-74 (2015).

2. Chen, F., et al. Neoantigen identification strategies enable personalized immunotherapy in refractory solid tumors. J Clin Invest 129, 2056-2070 (2019).

3. Hilf, N., et al. Actively personalized vaccination trial for newly diagnosed glioblastoma.

Nature 565, 240-245 (2019).

4. Keskin, D.B., et al. Neoantigen vaccine generates intratumoral T cell responses in phase Ib glioblastoma trial. Nature 565, 234-239 (2019).

5. Ott, P.A., et al. An immunogenic personal neoantigen vaccine for patients with melanoma. Nature 547, 217-221 (2017).

6. Peres, C., et al. Preclinical models and technologies to advance nanovaccine development. Adv Drug Deliv Rev 172, 148-182 (2021).

7. Irvine, D.J. \& Dane, E.L. Enhancing cancer immunotherapy with nanomedicine. Nat Rev Immunol 20, 321-334 (2020).

8. Moon, J.J., Huang, B. \& Irvine, D.J. Engineering nano- and microparticles to tune immunity. Adv Mater 24, 3724-3746 (2012).

9. $\mathrm{Xu}, \mathrm{J} .$, et al. A general strategy towards personalized nanovaccines based on fluoropolymers for post-surgical cancer immunotherapy. Nat Nanotechnol 15, 1043-1052 (2020).

10. Zong, L., Abe, M., Seto, Y. \& Ji, J. The challenge of screening for early gastric cancer in China. Lancet 388, 2606 (2016). 
11. Zhang, X., et al. Perioperative or postoperative adjuvant oxaliplatin with S-1 versus adjuvant oxaliplatin with capecitabine in patients with locally advanced gastric or gastro-oesophageal junction adenocarcinoma undergoing D2 gastrectomy (RESOLVE): an open-label, superiority and non-inferiority, phase 3 randomised controlled trial. Lancet Oncol 22, 1081-1092 (2021).

12. Tsuchiya, T., et al. A randomized phase II study of S-1 monotherapy versus cisplatin with vinorelbine for completely resected stage II/IIIA non-small cell lung cancer: rationale and study protocol design for the LOGIK1702 study. BMC Cancer 21, 249 (2021).

13. Noh, S.H., et al. Adjuvant capecitabine plus oxaliplatin for gastric cancer after D2 gastrectomy (CLASSIC): 5-year follow-up of an open-label, randomised phase 3 trial. Lancet Oncol 15, 1389-1396 (2014).

14. Kang, Y.K., et al. Nivolumab in patients with advanced gastric or gastro-oesophageal junction cancer refractory to, or intolerant of, at least two previous chemotherapy regimens (ONO-4538-12, ATTRACTION-2): a randomised, double-blind, placebo-controlled, phase 3 trial. Lancet 390, 2461-2471 (2017).

15. Pollack, K.E., et al. Incomplete Freund's adjuvant reduces arginase and enhances Th1 dominance, TLR signaling and CD40 ligand expression in the vaccine site microenvironment. J Immunother Cancer 8(2020).

16 Carroll, C.S., et al.Simple and effective bacterial-based intratumoral cancer immunotherapy. J Immunother Cancer 9(2021).

17. Fang, Y., et al. A Pan-cancer Clinical Study of Personalized Neoantigen Vaccine Monotherapy in Treating Patients with Various Types of Advanced Solid Tumors. Clin Cancer Res 26, 4511-4520 (2020). 
18. Ding, Z., et al. Personalized neoantigen pulsed dendritic cell vaccine for advanced lung cancer. Signal Transduct Target Ther 6, 26 (2021).

19. Zeng, Y., et al. Personalized neoantigen-based immunotherapy for advanced collecting duct carcinoma: case report. J Immunother Cancer 8(2020).

20. Hegde, P.S. \& Chen, D.S. Top 10 Challenges in Cancer Immunotherapy. Immunity 52, 17-35 (2020).

21 O'Donnell, J.S., Teng, M.W.L. \& Smyth, M.J. Cancer immunoediting and resistance to T cell-based immunotherapy. Nat Rev Clin Oncol 16, 151-167 (2019). Sagiv-Barfi, I., et al. Eradication of spontaneous malignancy by local immunotherapy. Sci Transl Med 10(2018). Ajani, J. A., et al. Gastric Cancer, Version 3.2016, NCCN Clinical Practice Guidelines in Oncology. J Natl Compr Canc Netw 14, 1286-1312 (2016). Wang, F.H., et al. The Chinese Society of Clinical Oncology (CSCO): Clinical guidelines for the diagnosis and treatment of gastric cancer, 2021. Cancer Commun (Lond) 41, 747-795 (2021).

25. Mita, K., et al. Adjuvant Chemotherapy with S-1 plus Cisplatin for Patients with Stage III Gastric Cancer After Curative Resection. Anticancer Res 37, 1329-1333 (2017).

21. Saxena, M., van der Burg, S.H., Melief, C.J.M. \& Bhardwaj, N. Therapeutic cancer vaccines. Nat Rev Cancer 21, 360-378 (2021).

22. Malonis, R.J., Lai, J.R. \& Vergnolle, O. Peptide-Based Vaccines: Current Progress and Future Challenges. Chem Rev 120, 3210-3229 (2020).

23. Sahin, U. \& Tureci, O. Personalized vaccines for cancer immunotherapy. Science 359, $1355-1360$ (2018). 
24. Hu, Z., Ott, P.A. \& Wu, C.J. Towards personalized, tumour-specific, therapeutic vaccines for cancer. Nat Rev Immunol 18, 168-182 (2018).

30. Dhodapkar, M.V., et al. Induction of antigen-specific immunity with a vaccine targeting NY-ESO-1 to the dendritic cell receptor DEC-205. Sci Transl Med 6, 232ra251 (2014).

31 Simpson, A., Caballero, O., Jungbluth, A., Chen, Y. \& Old, L. Cancer/testis antigens, gametogenesis and cancer. Nat Rev Cancer 5, 615-625 (2005).

32. Cappellano, G., Abreu, H., Casale, C., Dianzani, U. \& Chiocchetti, A. Nano-Microparticle Platforms in Developing Next-Generation Vaccines. Vaccines (Basel) 9(2021).

33. Blass, E. \& Ott, P.A. Advances in the development of personalized neoantigen-based therapeutic cancer vaccines. Nat Rev Clin Oncol 18, 215-229 (2021).

$34 \mathrm{Ni}, \mathrm{Q}$., et al. A bi-adjuvant nanovaccine that potentiates immunogenicity of neoantigen for combination immunotherapy of colorectal cancer. Sci Adv 6, eaaw6071 (2020). Skakuj, K., et al. Conjugation Chemistry-Dependent T-Cell Activation with Spherical Nucleic Acids. J Am Chem Soc 140, 1227-1230 (2018).

36. Callmann, C.E., et al. Tumor cell lysate-loaded immunostimulatory spherical nucleic acids as therapeutics for triple-negative breast cancer. Proc Natl Acad Sci U S A 117, 17543-17550 (2020).

37. Hu, Z.T., et al. Personal neoantigen vaccines induce persistent memory $\mathrm{T}$ cell responses and epitope spreading in patients with melanoma.Nat Med 27, 515-525 (2021).

38. Smyth, E.C., Gambardella, V., Cervantes, A. \& Fleitas, T. Checkpoint inhibitors for gastroesophageal cancers: Dissecting heterogeneity to better understand their role in first line and adjuvant therapy. Ann Oncol (2021). 
39. Zhou, K.I., et al. Spatial and Temporal Heterogeneity of PD-L1 Expression and Tumor Mutational Burden in Gastroesophageal Adenocarcinoma at Baseline Diagnosis and after Chemotherapy. Clin Cancer Res 26, 6453-6463 (2020).

40. Kwon, M., et al. Determinants of Response and Intrinsic Resistance to PD-1 Blockade in Microsatellite Instability-High Gastric Cancer. Cancer Discov (2021).

41. Guo, X.G., et al. CNSA: a data repository for archiving omics data. Database (Oxford), baaa055 (2020).

42. Chen, F.Z., et al. CNGBdb: China National GeneBank DataBase. Yi Chuan 42, 799-809 (2020). 


\section{Figure legends}

Fig. 1 Neoantigen nanovaccines accumulate in lymph nodes (LNs) and activate

BMDCs in vivo. a, ${ }^{1} \mathrm{H}$ nuclear magnetic resonance (NMR) spectra of DSPE-PEG 2000 -NHS, DSPE-PEG 2000 -peptide and peptide. The insert in DSPE-PEG 2000 -peptide shows the proton signal from methyl groups in antigen peptide, indicating portions of peptide were successfully conjugated into the copolymers. $\mathbf{b}$, Transmission electron microscope image of nanovaccines with a monolayer spherical morphology (20-30 nm diameter ). Scale bar, $200 \mathrm{~nm}$. c,d,e, Neoantigens of MFC tumor cells, which were defined as MFC-1 or MFC-2 to MFC-9, were labeled with Cy5. A total of 615 mice were injected subcutaneously with free Cy5-labeled neoantigens or nanovaccines at the tail base, respectively $(n=3)$. c, Near-infrared images of the biodistribution of PNVAC in mice at 2, 24 and 48 hours after subcutaneous injection. d, Near-infrared images of inguinal LNs acquired after 48 hours. e, Fluorescence semi-quantification of peptide accumulation in LNs at different time points following subcutaneous injection of free vaccine or nanovaccine $(n=3)$. $\mathbf{f}$, Localization of $\mathrm{CD}^{+} \mathrm{T}$ cells (gray) or $\mathrm{CD}^{+1} \mathrm{c}^{+} \mathrm{DCs}$ (red) and FITC-labeled nanovaccines (green) at $48 \mathrm{~h}$ after subcutaneous injection in LNs. g, IVIS quantification of peptide concentration in blood from 615 mice after subcutaneous immunization with $100 \mu \mathrm{g}, 300 \mu \mathrm{g}$ or $900 \mu \mathrm{g}$ nanovaccines $(\mathrm{n}=3) . \quad \mathrm{i}$, Mouse bone marrow derived cells (BMDCs) were incubated with NS (normal saline), free vaccines and nanovaccines (MFC-1 or MFC-6) for $48 \mathrm{~h}$, and $\mathrm{CD} 80^{+} \mathrm{CD} 86^{+} \mathrm{DCs}$ were quantified using flow cytometry to assess DCs maturation. Data was analyzed to 
compare NS with free vaccines using one-way (i) or two-way ANOVA (e) with Tukey's HSD multiple comparison post hoc test. $* P<0.05$, ** $P<0.01, * * * P<$ 0.001 .

Fig. 2 Neoantigen nanovaccines inhibit tumor growth and prolong survival time.

a, Treatment schema. A total of $2 \times 10^{6} \mathrm{MFC}$ cells were subcutaneously injected into 615 mice. The established tumors were removed when the tumor volume reached 100 $\mathrm{mm}^{3}$. Three days after surgery, mice were randomly divided into 6 groups and injected subcutaneously with NS, blank vehicle, free vaccines, nanovaccines, anti-PD-1 (5 mg kg-1, injected intraperitoneally) or nanovaccines + anti-PD-1 (5 mg $\mathrm{kg}^{-1}$ injected intraperitoneally). The mice were immunized with nanovaccines $(20 \mu \mathrm{g}$ each antigen) five times on the indicated days. b, MRI images of representative mice were acquired on day 25 and showed tumor regression after PNVAC vaccination. The tumor of each mouse is circled. Shown are Kaplan-Meier survival curves (c), average tumor growth curves (d) and individual MFC tumor growth curves (e) in different groups $(n=6)$. Data was analyzed using two-way ANOVA (c) with Tukey's HSD multiple comparison post hoc test or Log-rank (Mantel-Cox) test (d). ${ }^{*} P<0.05$, ${ }^{* *} P$ $<0.01, * * * P<0.001$.

Fig. 3 Neoantigen nanovaccines promote DCs mature and elicit specific $T$ cell responses. a, b, Mouse splenocytes were isolated one week after the last vaccination. Proportions of mature DCs $\left(\mathrm{CD} 11 \mathrm{c}^{+} \mathrm{CD} 80^{+} \mathrm{CD} 86^{+}\right)$and neoantigen (MFC-1)-specific 
$\mathrm{T}$ cells $\left(\mathrm{CD}^{+} \mathrm{CD}^{+} \mathrm{MFC}-3-\mathrm{H}_{2} \mathrm{~K}^{\mathrm{k}+}\right)$ in mouse splenocytes analyzed using flow cytometry. c, IFN- $\gamma$ secretion of splenocytes which were re-stimulated with 9 neoantigens of MFC cells. d, MFC tumor bearing mice in nanovaccine group that achieved a complete response were subcutaneously re-challenged with B16F10 melanoma tumor cells $\left(2 \times 10^{5}\right)$ in the left side of abdomen and MFC gastric cancer cells $\left(2 \times 10^{6}\right)$ in the right side of abdomen 40 days later. Shown are tumor growth curves for each mouse following the re-challenge $(n=5)$. Pictures of representative mice were taken on day 20 after re-challenge and tumors were circled. e, Proportions of effector memory $\mathrm{T}$ cells (TEM, $\left.\mathrm{CD} 3{ }^{+} \mathrm{CD} 8{ }^{+} \mathrm{CD} 44^{+} \mathrm{CD} 62 \mathrm{~L}^{-}\right)$in mouse splenocytes analyzed using flow cytometry. f, Pathways with significant differential expression between NS and nanovaccines groups are presented based on the gene hit size using RNA-seq. Gene expressions were clustered using $\log _{10}(\mathrm{FKPM}+1)$ and $\mathrm{GO}$ enrichment analysis. g, h, Differential gene expressions in MHC I and II pathway (g) and type I IFN responses (h). Data was analyzed using one-way (a, b, c, e) with Tukey's HSD multiple comparison post hoc test. ** $P<0.01, * * * P<0.001$.

Fig. 4 Generation of an individualized, multiepitope neoantigen nanovaccine in patients with G/GEJ cancer. a, Tumor somatic mutations of gastric cancer were identified using WES and genes expression was confirmed using RNA-seq. CTAs expression was tested using immunochemical staining. Immunized neoantigens were identified on the basis of HLA binding affinity predictions and manufactured into nanovacines. The prepared nanovaccines and adjuvant Montanid ISA 51 were 
injected subcutaneously at 4 injection sites. b, Clinical outcomes of 29 vaccinated G/GEJ cancer patients after surgery until the disease was progressed or the cut-off date, Dec 31, 2020. Asterisk represents patients with progressed diseases and arrow represents disease free patients.). c, Ultrasound images of lymph nodes after PNVAC injection. d, e, Summary of T cells immune response to PNVAC (d: ex vivo; e: in vitro stimulation).

Fig. 5 Nanovaccine induces multifunctional circulating $\mathrm{CD4}^{+}$and $\mathrm{CD8}^{+} \mathrm{T}$ cell responses in gastric cancer patients. a, IFN- $\gamma$ ELISPOT of patient 035 PBMCs stimulated with individualized peptides to assess the neoantigen-specific $\mathrm{T}$ cell responses. No peptide media and phytohemagglutinin (PHA) were used as negative control and positive control, respectively. b, ICS of PBMCs after neoantigens stimulation. PBMCs were pre-gated based on $\mathrm{CD}^{+}$and $\mathrm{CD}^{+} \mathrm{T}$ cells. Cytokine-producing neoantigen-specific $\mathrm{T}$ cells expressed CD45RO, demonstrating an antigen-experienced memory $\mathrm{T}$ cell phenotype. c, In vitro measurement of intracellular IFN- $\gamma^{+}, \mathrm{TNF}-\alpha^{+}$and $\mathrm{IL}-2^{+} \mathrm{CD} 4^{+} \mathrm{T}$ cells in PBMCs from patient 035 who was treated with individualized peptides. d, In vitro measurement of intracellular IFN $-\gamma^{+}, \mathrm{TNF}-\alpha^{+}$and IL-2 ${ }^{+} \mathrm{CD}^{+} \mathrm{T}$ cells in patient 035 PBMCs which was stimulated with individualized peptides. e, Median percentage of cytokine production across 21 patients by $\mathrm{CD}^{+} \mathrm{T}$ cells stimulated in vitro with immunized peptides. Data was analyzed by paired two-tailed Student's $t$-test. $* * P<0.01$. f, Nanovaccines induced persistence of immune responses, as indicated by level of IFN- $\gamma$ in PBMCs at week 
52 after nanovaccines therapy among 12 patients. The number of responsive peptides for each patient is represented as orange stacked column at week 52 and the blue stacked column represents the number of no-responsive peptides. g, The PBMCs of patient 056 at different time points were restimulated with immunized peptides overnight. Then the IFN- $\gamma$ concentration in culture supernatant was measured by IFN- $\gamma$ CBA assay. $\mathbf{h}$, ICS of IFN- $\gamma$ and TNF- $\alpha$ cytokine-producing CD4 ${ }^{+} \mathrm{T}$ cells in PBMCs from patient 056 after one round pre-stimulation at the specific time point. $\mathbf{i}$, Pie charts show that total $\mathrm{CD}^{+} \mathrm{T}$ cell responses positive for 1 or 2 cytokines (IFN- $\gamma$, TNF- $\alpha$ or IL-2) at week 18 and week 110 after vaccination initiation in patient 002. Bar graphs show the absolute frequencies of ASP pool-reactive $\mathrm{CD}^{+} \mathrm{T}$ cells producing 1 or 2 cytokines.

Fig. 6 Clinical and immune responses to anti-PD-1 blockade alone in patient 023 who relapsed after personalized PNVAC. a, In patient 023 , PET/CT scan revealed inflammatory uptake at the vaccinated sites. b, The neoantigen-specific $\mathrm{T}$ cell responses were enhanced after PD-1 blockade in patient 023 who relapsed after vaccination. c, Computed tomography (CT) scans showed that after 3 doses of anti-PD-1 blockade immunotherapy, multiple metastatic lymph nodes shrank, but ascites appeared. d, Comparison of somatic mutations between primary tumor and relapsed lesion detected using WES. e, Comparison of gene mutations corresponding to immunized neoantigens between primary tumor and progressed lesion in patient 023. 
Supplementary Fig.1 Characterization of neoantigen-based nanovaccines. a, Molecular weight characterization of DSPE-PEG 2000 -Peptide analyzed using MALDI-TOF mass spectrometry. $\mathbf{b}$, Size of nanovaccines analyzed using a Malvern Zetasizer. c, Size, polydispersity index (PDI), encapsulation efficiency, drug loading content and zeta potential of nanovaccines $(n=9)$. d, Size and PDI of nanovaccines were kept stable when nanovaccines were incubated in normal saline for $96 \mathrm{~h}$ at $4^{\circ} \mathrm{C}$ or $37^{\circ} \mathrm{C}(\mathrm{n}=3$, mean \pm s.d $)$.

Supplementary Fig. 2 Neoantigen-based nanovaccines activate BMDCs. a,b, Statistical data (a) and representative flow cytometry analysis (b) showed nanovaccines induced more mature $\mathrm{DCs}\left(\mathrm{CD} 80^{+} \mathrm{CD} 86^{+} \mathrm{CD} 11 \mathrm{c}^{+} \mathrm{DCs}\right)$ than normal saline (NS), and free neoantigens after in vitro stimulation BMDCs for $48 \mathrm{~h}(\mathrm{n}=4)$. Data were analyzed using one-way ANOVA. ${ }^{*} P<0.05, * * P<0.01, * * * P<0.001$.

Supplementary Fig. 3 Safety of neoantigen-based nanovaccines. a,b, A total of 615 mice were randomly divided into 5 groups and subcutaneously injected with NS and 4 doses of nanovaccines $(0.75 \mathrm{mg} / \mathrm{kg}, 3.75 \mathrm{mg} / \mathrm{kg}, 7.5 \mathrm{mg} / \mathrm{kg}$ and $15 \mathrm{mg} / \mathrm{kg})$. Shown are hematology indices $(\mathrm{n}=8)$. Data was analyzed using one-way ANOVA. c,d, MFC cells $\left(2 \times 10^{6}\right)$ were subcutaneously injected into 615 mice. The established tumors were removed when the tumor volume reached $\sim 100 \mathrm{~mm}^{3}$. Three days after surgery, the tumor-bearing mice were randomly divided into 6 groups and injected

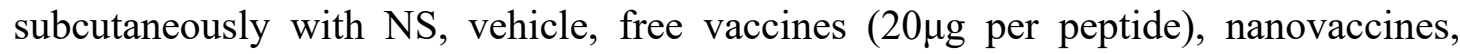


anti-PD-1 (0.5 mg/kg, intraperitoneally injected $)$ or nanovaccines + anti-PD-1 (intraperitoneally injected). c, Hematoxylin and eosin staining of tissues sections from main organs, including heart, lung, liver and kidney (scale bar, $100 \mu \mathrm{m}$ ). (D) Body weights of MFC tumor bearing mice in each group $(n=6)$.

Supplementary Fig. 4 Neoantigen nanovaccines induce DCs mature and stimulate specific $\mathbf{T}$ cell responses. a, Proportions of $\mathrm{CD} 80^{+} \mathrm{CD} 86^{+} \mathrm{CD} 11 \mathrm{c}^{+} \mathrm{DCs}$ in lymph nodes were analyzed using flow cytometry on day 7 after the last vaccination. b, Proportions of neoantigen-specific T cells $\left(\mathrm{CD} 3^{+} \mathrm{CD}^{+} \mathrm{MFC}-\mathrm{H}_{2} \mathrm{~K}^{\mathrm{k}+}\right)$ in spleens were analyzed using flow cytometry one week after the last treatment (MFC-2 to MFC-9). c, Splenocytes from mice in each group were isolated and incubated with CFSE-labeled MFC cancer cells at effector-to-target ratios (E: T) of 12.5:1, 25:1 and 50:1. PI was added after 6 hours incubation and the percentage of dead cells $\left(\mathrm{CFSE}^{+} \mathrm{PI}^{+}\right)$was analyzed using flow cytometry. d, Nanovaccines increased PD- $1^{+}$ $\mathrm{CD}^{+} \mathrm{T}$ cells in tumors. Data were analyzed by one-way ANOVA. $* P<0.05,{ }^{*} * P<$ $0.01, * * * P<0.001$

Supplementary Fig. 5, 6 Demonstration of epitope spreading. IFN- $\gamma$ secretion by PBMCs pre- and post- vaccination followed by overnight incubation with individual peptides or no peptide was assessed using ex vivo CBA assay.

Supplementary Fig. 7 Demonstration of epitope spreading. IFN- $\gamma$ secretion by PBMCs pre- and post- vaccination followed by 11-day stimulation with individual 
peptides or no peptide was assessed using in vitro CBA assay.

Supplementary Fig. 8 a-c, Frequencies of cytokine-producing $\mathrm{CD}^{+}$and $\mathrm{CD}^{+} \mathrm{T}$ cells in response to neoantigens in 21 patients, as measured by ICS after in vitro stimulation of PBMCs with the immunizing peptides before and after PNVAC treatment. Data was analyzed by paired two-tailed Student's $t$-test.

Supplementary Fig. 9 Pie charts for $\mathrm{CD4}^{+} \mathrm{T}$ cell responses in patient 002 . Total $\mathrm{CD}^{+} \mathrm{T}$ cell responses positive for one or two cytokines at week 18 and week 110 post vaccination. Bar graphs show absolute frequencies of immunized neoepitopes-specific $\mathrm{CD}^{+} \mathrm{T}$ cells secreting one or two cytokines.

Supplementary Fig. 10 Gating strategy. For all stainings, a lymphocyte gate was drawn using FSC/SSC gating first. For intracellular cytokine staining, gating was determined based on negative control (no peptide stimulation).

Supplementary Table 1 Clinical characteristics of patients.

Supplementary Table 2 Individualized neoantigen for each patient.

Supplementary Table 3 Summary of adverse events.

Supplementary Table 4 Quality control for WES and RNA-seq.

The supplement file. The clinical trial protocol. 

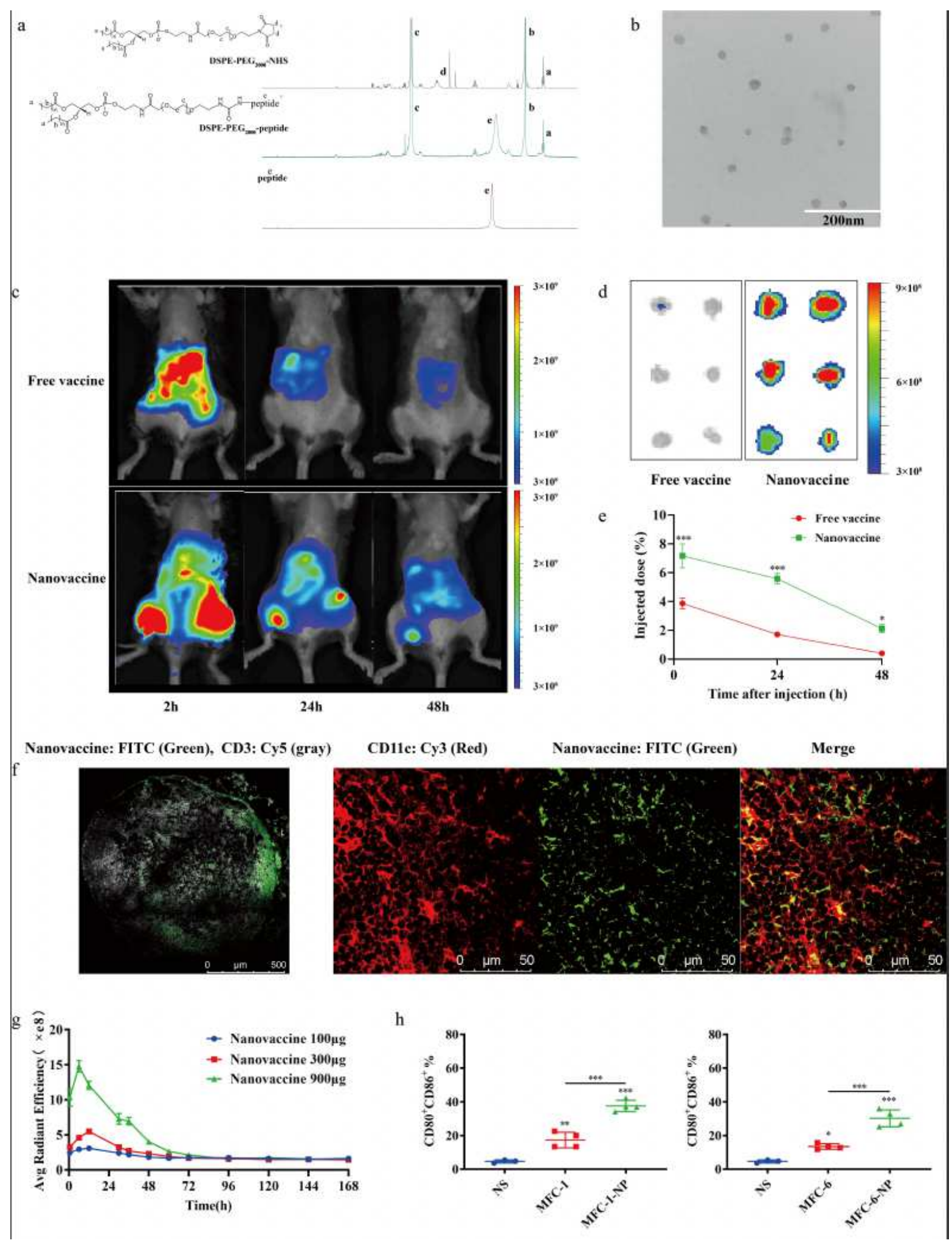

\section{Figure 1}

Neoantigen nanovaccines accumulate in lymph nodes (LNs) and activate BMDCs in vivo. a, $1 \mathrm{H}$ nuclear magnetic resonance (NMR) spectra of DSPE-PEG2000-NHS, DSPE-PEG2000-peptide and peptide. The insert in DSPE-PEG2000-peptide shows the proton signal from methyl groups in antigen peptide, 
indicating portions of peptide were successfully conjugated into the copolymers. b, Transmission electron microscope image of nanovaccines with a monolayer spherical morphology (20-30 nm diameter ). Scale bar, $200 \mathrm{~nm}$. c,d,e, Neoantigens of MFC tumor cells, which were defined as MFC-1 or MFC-2 to MFC-9, were labeled with Cy5. A total of 615 mice were injected subcutaneously with free Cy5-labeled neoantigens or nanovaccines at the tail base, respectively $(n=3)$. c, Near-infrared images of the biodistribution of PNVAC in mice at 2, 24 and 48 hours after subcutaneous injection. d, Near-infrared images of inguinal LNs acquired after 48 hours. e, Fluorescence semi-quantification of peptide accumulation in LNs at different time points following subcutaneous injection of free vaccine or nanovaccine $(n=3)$. f, Localization of CD3+ T cells (gray) or CD11c+ DCs (red) and FITC-labeled nanovaccines (green) at $48 \mathrm{~h}$ after subcutaneous injection in LNs. g, IVIS quantification of peptide concentration in blood from 615 mice after subcutaneous immunization with $100 \mu \mathrm{g}, 300 \mu \mathrm{g}$ or $900 \mu \mathrm{g}$ nanovaccines $(n=3)$. i, Mouse bone marrow derived cells (BMDCs) were incubated with NS (normal saline), free vaccines and nanovaccines (MFC-1 or MFC-6) for $48 \mathrm{~h}$, and CD80+CD86+DCs were quantified using flow cytometry to assess DCs maturation. Data was analyzed to compare NS with free vaccines using one-way (i) or two-way ANOVA (e) with Tukey's HSD multiple comparison post hoc test. * $\mathrm{P}<0.05,{ }^{* *} \mathrm{P}<0.01, * * * \mathrm{P}<0.001$. 
a

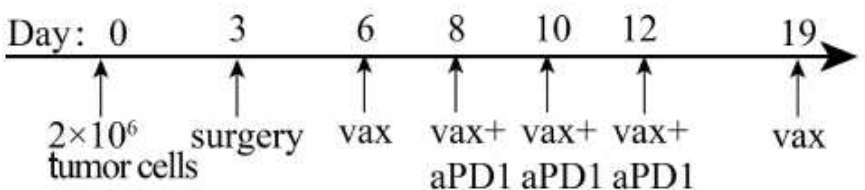

c

+ NS - Vehicle + Free vaccine

+ Nanovaccine + aPD-1 - Nanovaccine+aPD-1

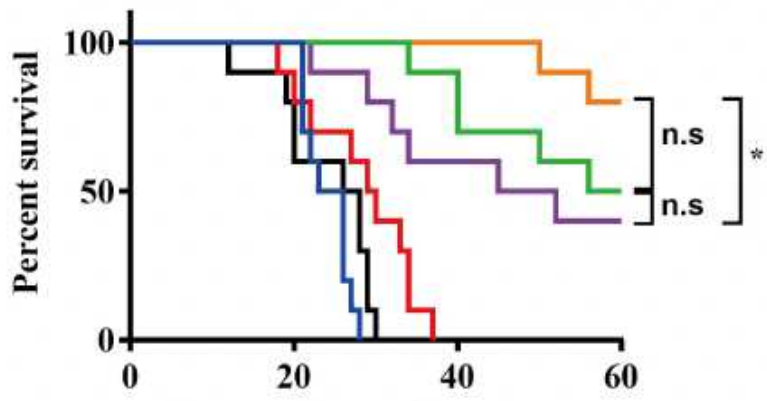

Days post tumor inoculation
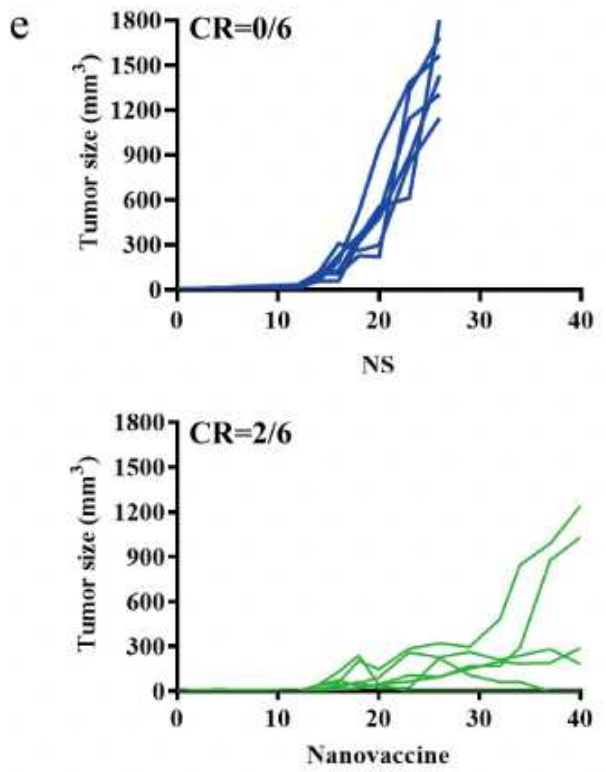

b

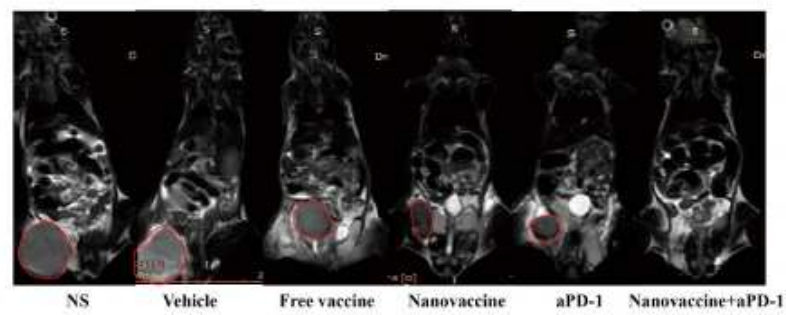

d

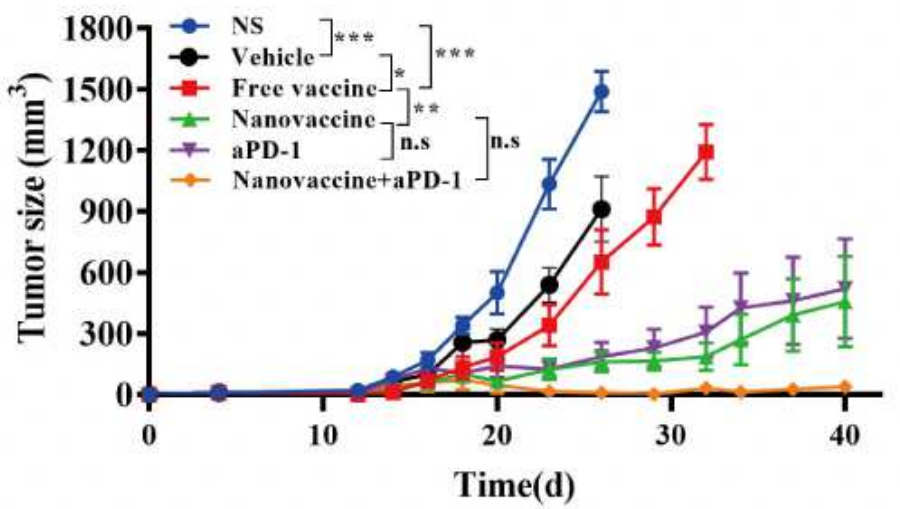

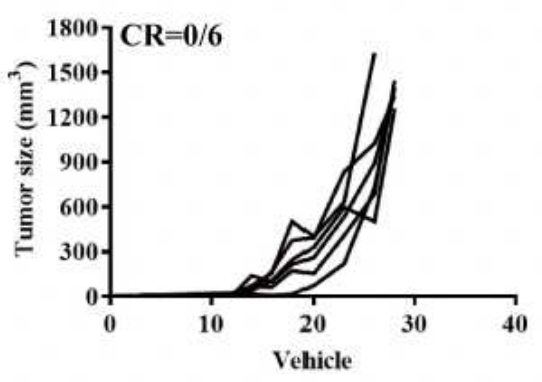

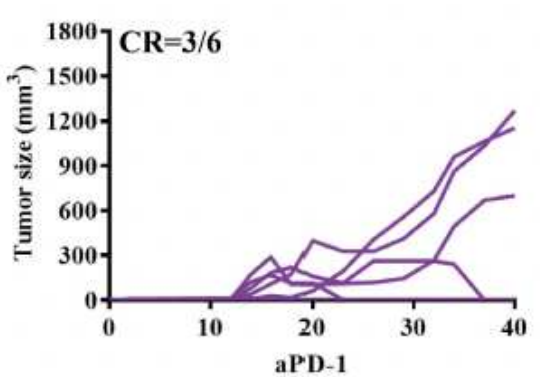

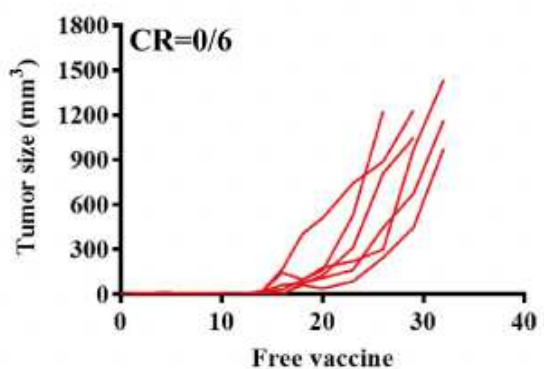

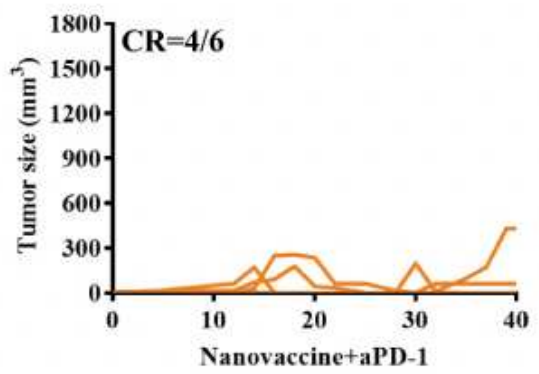

Figure 2

Neoantigen nanovaccines inhibit tumor growth and prolong survival time. a, Treatment schema. A total of $2 \times 106$ MFC cells were subcutaneously injected into 615 mice. The established tumors were removed when the tumor volume reached $100 \mathrm{~mm}$. Three days after surgery, mice were randomly divided into 6 groups and injected subcutaneously with NS, blank vehicle, free vaccines, nanovaccines, anti-PD-1 (5 mg kg-1, injected intraperitoneally) or nanovaccines + anti-PD-1 (5 mg kg-1 injected intraperitoneally). The

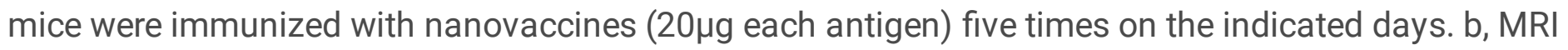
images of representative mice were acquired on day 25 and showed tumor regression after PNVAC vaccination. The tumor of each mouse is circled. Shown are Kaplan-Meier survival curves (c), average tumor growth curves (d) and individual MFC tumor growth curves (e) in different groups $(n=6)$. Data was 
analyzed using two-way ANOVA (c) with Tukey's HSD multiple comparison post hoc test or Log-rank (Mantel-Cox) test $(\mathrm{d}) .{ }^{*} \mathrm{P}<0.05$, ** $\mathrm{P}<0.01, * \star * P<0.001$.

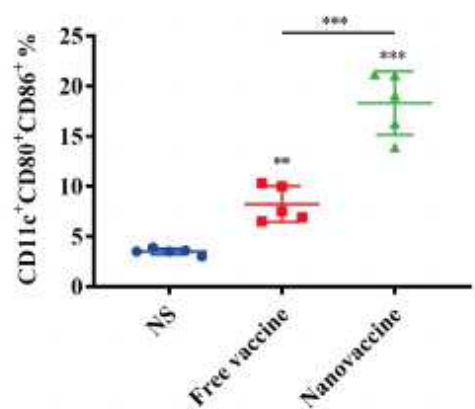

$\mathrm{c}$

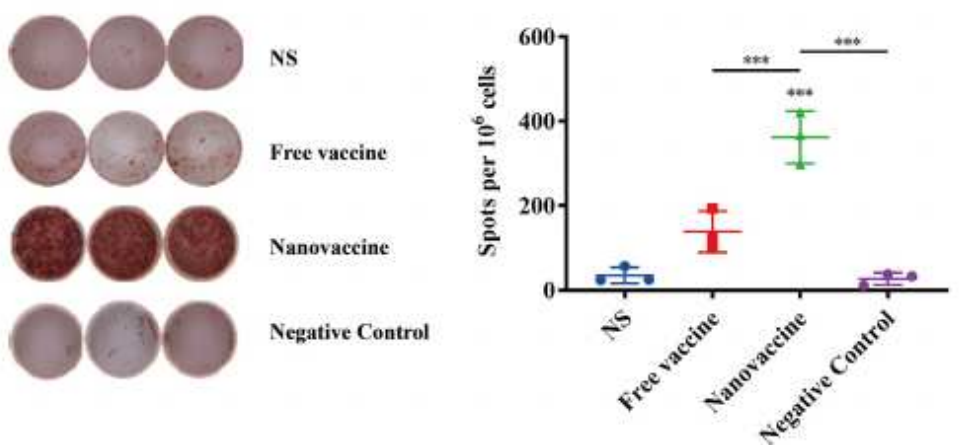

$\mathrm{e}$

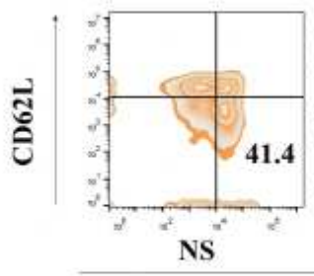

b

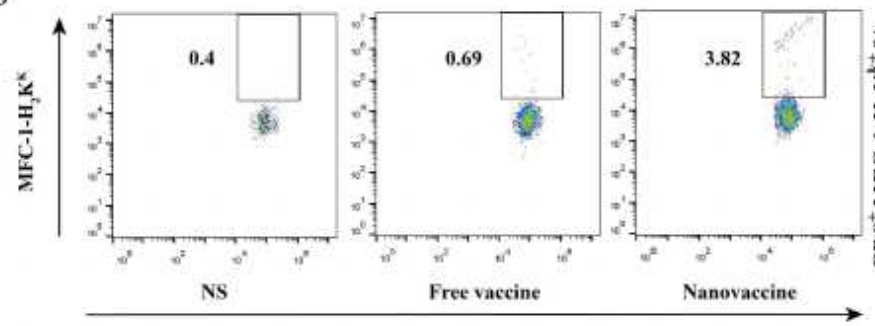

CD8 gate on $\mathrm{CD3}^{+} \mathrm{CD}^{+}$
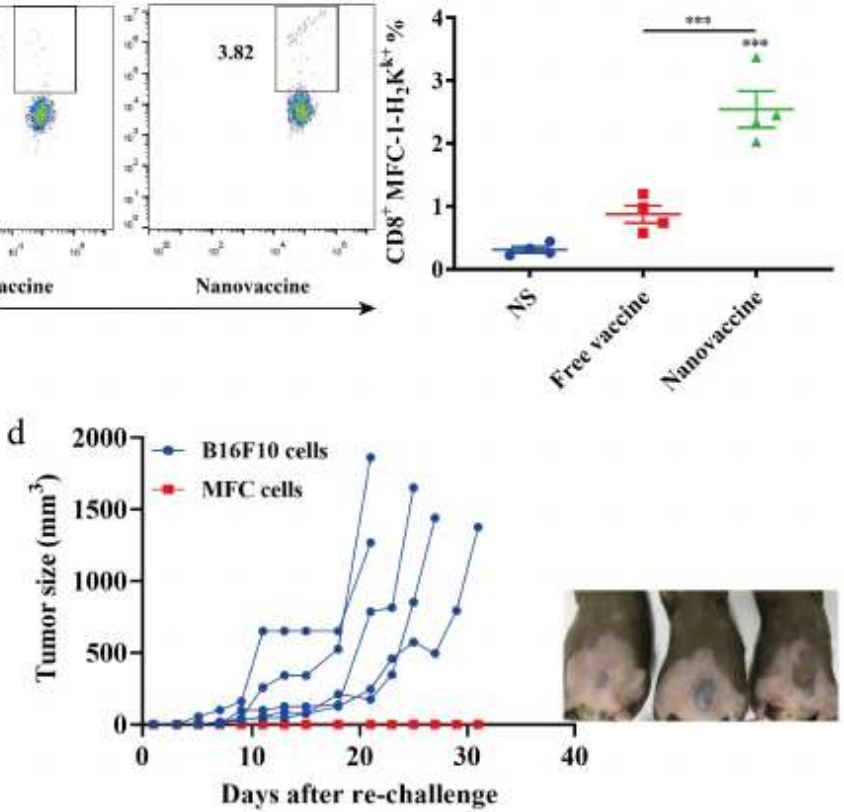

CD44 gate on CD8
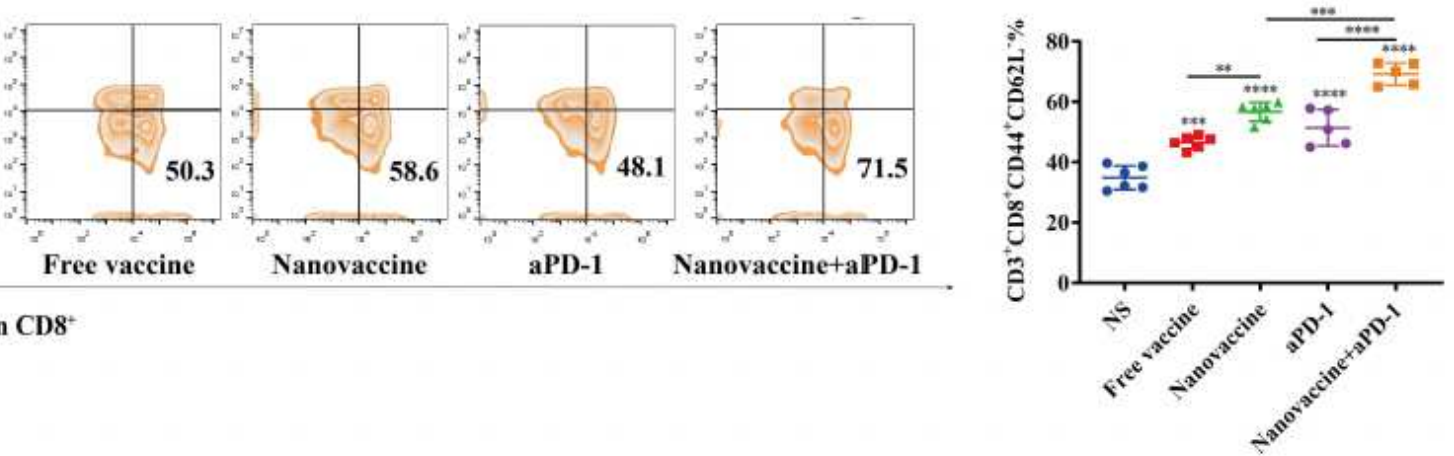

f
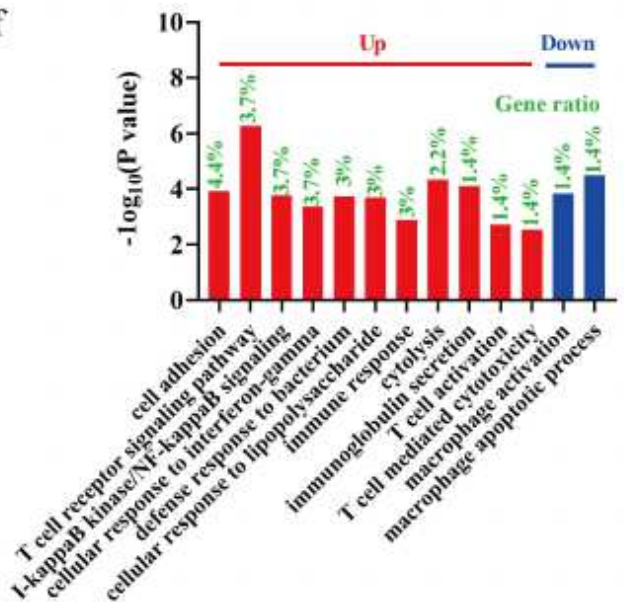
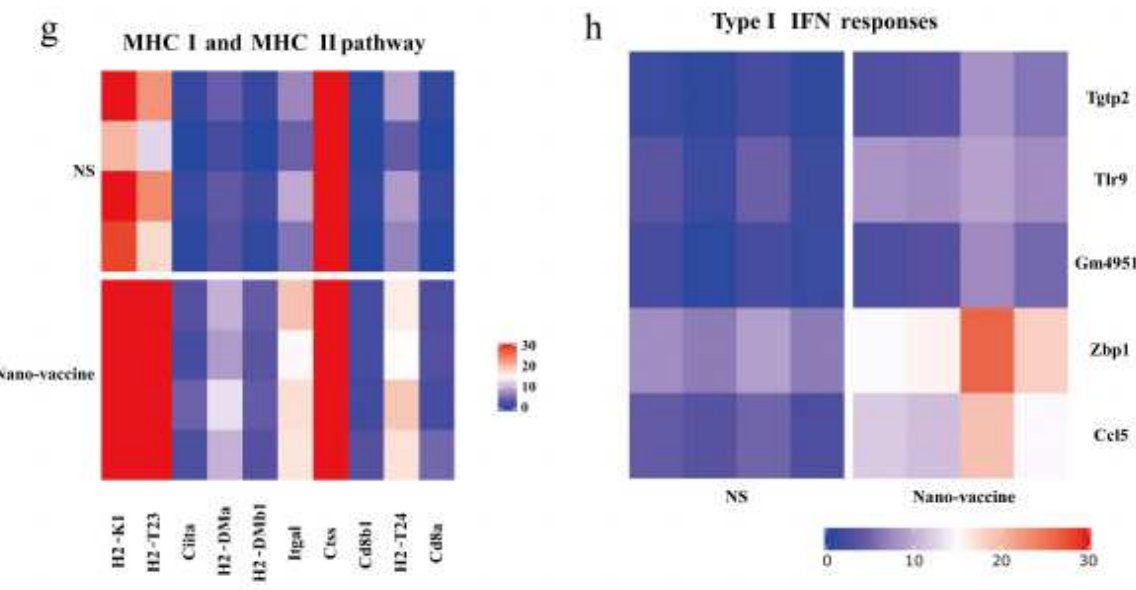

\section{Figure 3}

Neoantigen nanovaccines promote DCs mature and elicit specific T cell responses. a, b, Mouse splenocytes were isolated one week after the last vaccination. Proportions of mature DCs (CD11c+CD80+CD86+) and neoantigen (MFC-1)-specific T cells (CD3+CD8+MFC-3-H2Kk+) in mouse 
splenocytes analyzed using flow cytometry. c, IFN- $y$ secretion of splenocytes which were re-stimulated with 9 neoantigens of MFC cells. $d$, MFC tumor bearing mice in nanovaccine group that achieved a complete response were subcutaneously re-challenged with B16F10 melanoma tumor cells $(2 \times 105)$ in the left side of abdomen and MFC gastric cancer cells $(2 \times 106)$ in the right side of abdomen 40 days later. Shown are tumor growth curves for each mouse following the re-challenge $(n=5)$. Pictures of representative mice were taken on day 20 after re-challenge and tumors were circled. e, Proportions of effector memory $T$ cells (TEM, CD3+CD8+CD44+CD62L-) in mouse splenocytes analyzed using flow cytometry. $f$, Pathways with significant differential expression between NS and nanovaccines groups are presented based on the gene hit size using RNA-seq. Gene expressions were clustered using $\log 10(F K P M+1)$ and GO enrichment analysis. g, h, Differential gene expressions in MHC I and II pathway (g) and type I IFN responses (h). Data was analyzed using one-way ( $a, b, c, e)$ with Tukey's HSD multiple comparison post hoc test. ${ }^{* *} \mathrm{P}<0.01,{ }^{\star \star *} \mathrm{P}<0.001$. 
a

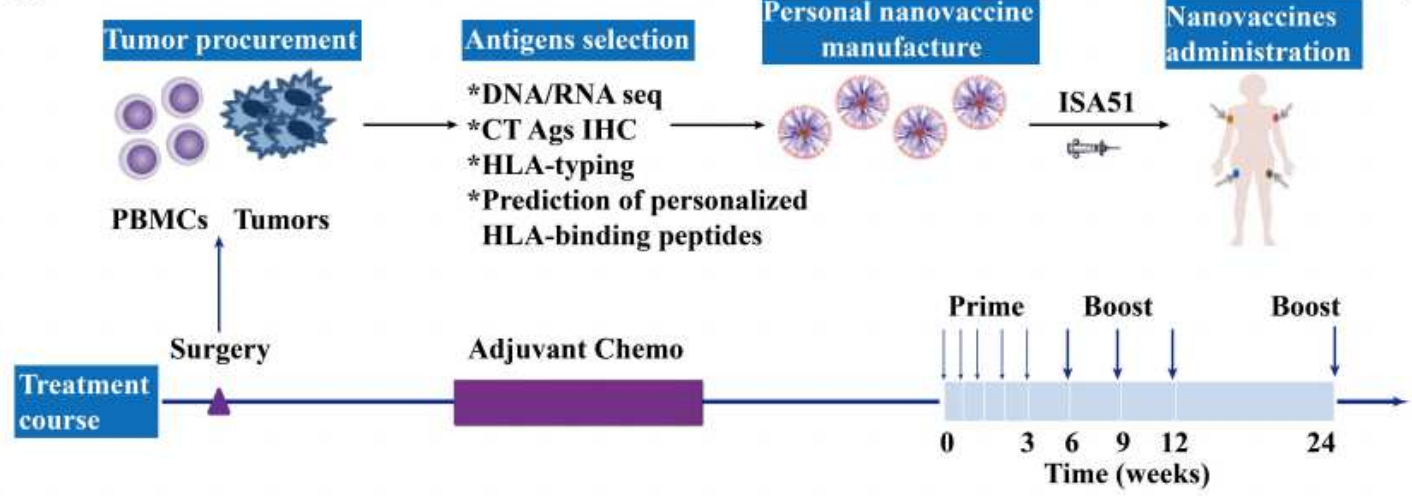

b

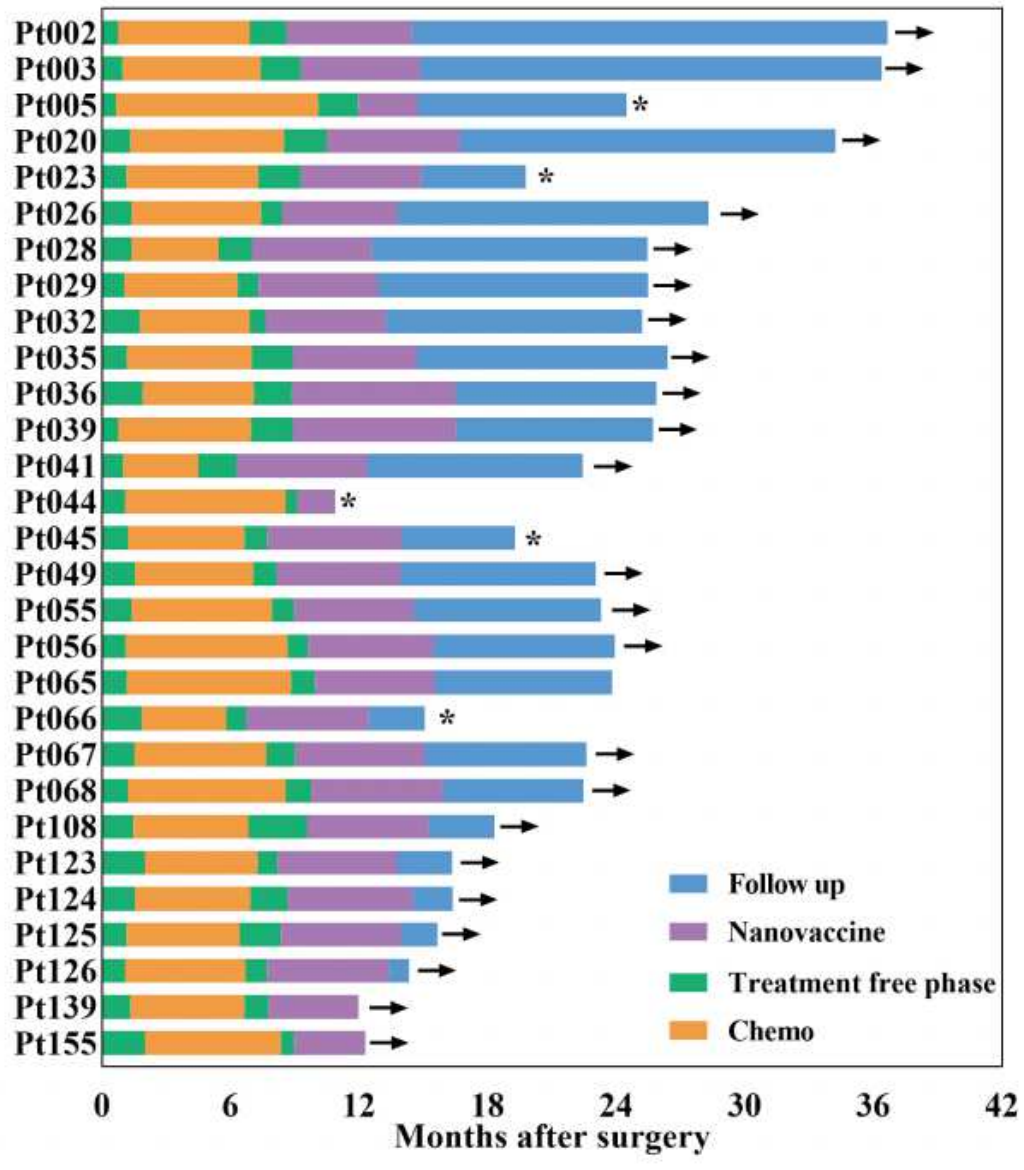

c Pt005

D2 after last vaccinization

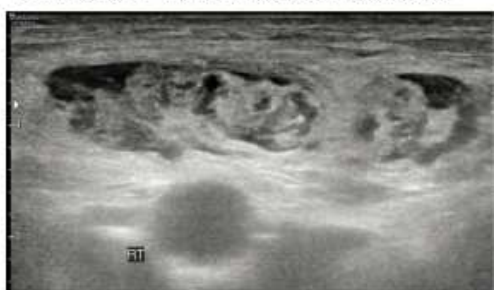

D490 after last vaccinization

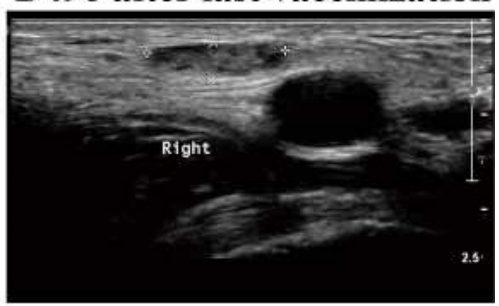

d ex vivo $\mathrm{T}$ cell responses

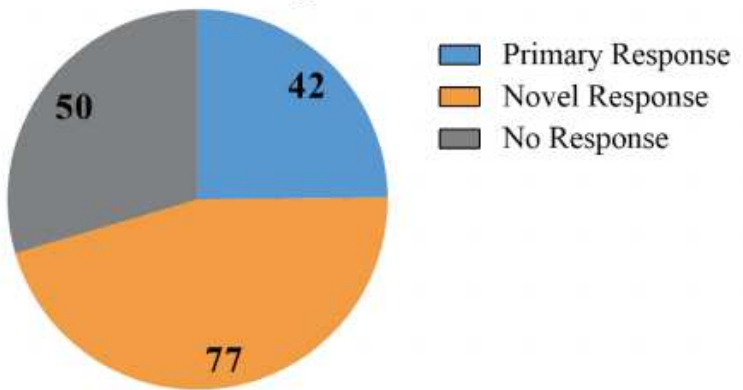

e in vitro $\mathrm{T}$ cell responses

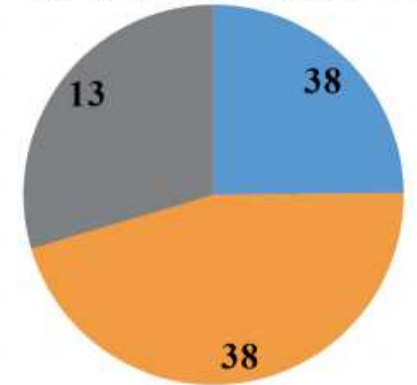

$\square$ Primary Response

$\square$ Novel Response

$\square$ No Response

\section{Figure 4}

Generation of an individualized, multiepitope neoantigen nanovaccine in patients with G/GEJ cancer. a, Tumor somatic mutations of gastric cancer were identified using WES and genes expression was confirmed using RNA-seq. CTAs expression was tested using immunochemical staining. Immunized neoantigens were identified on the basis of HLA binding affinity predictions and manufactured into nanovacines. The prepared nanovaccines and adjuvant Montanid ISA 51 were injected subcutaneously at 4 injection sites. b, Clinical outcomes of 29 vaccinated G/GEJ cancer patients after surgery until the disease was progressed or the cut-off date, Dec 31, 2020. Asterisk represents patients with progressed diseases and arrow represents disease free patients.). c, Ultrasound images of lymph nodes after PNVAC injection. $d$, e, Summary of T cells immune response to PNVAC (d: ex vivo; e: in vitro stimulation). 


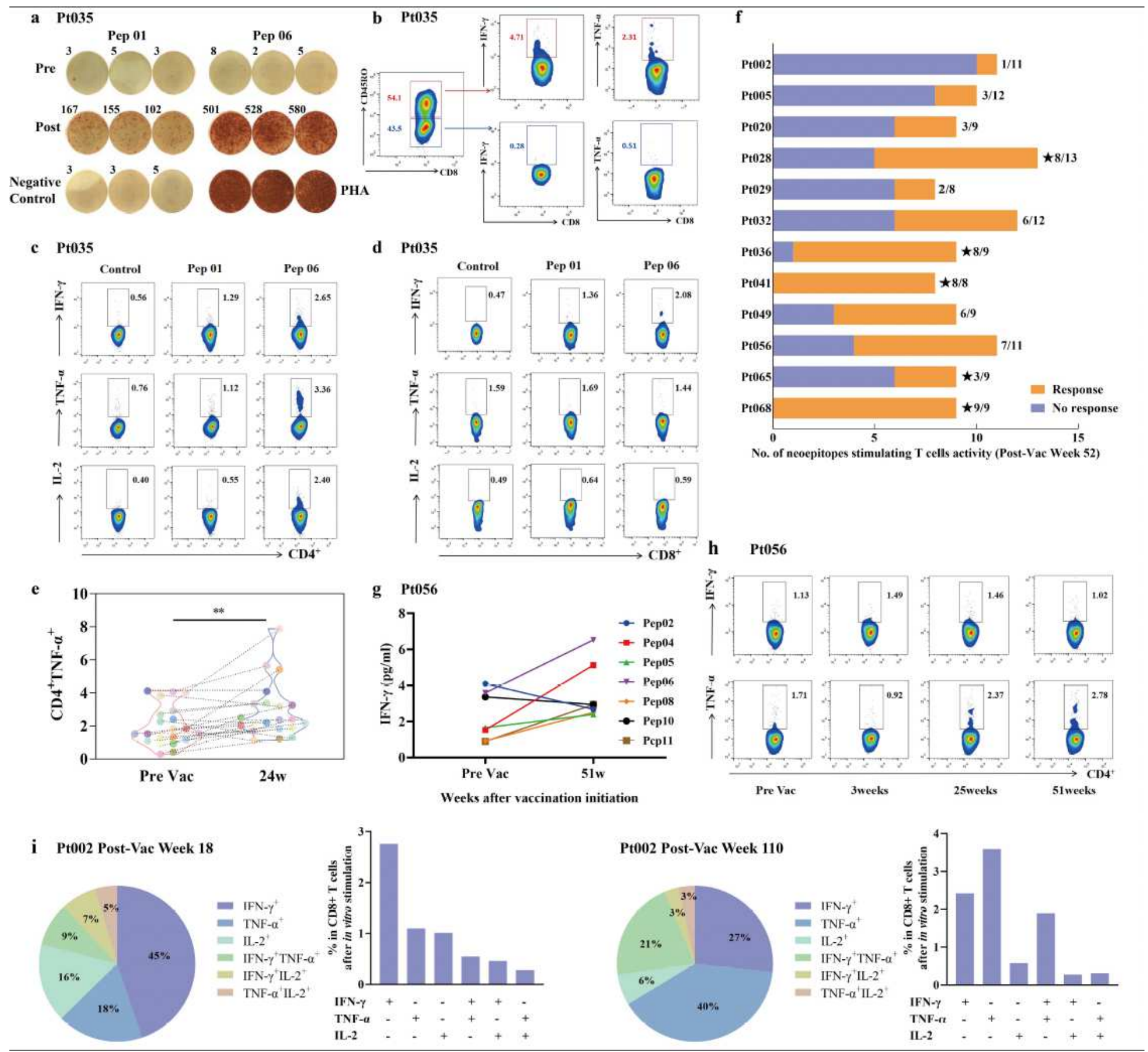

Figure 5

Nanovaccine induces multifunctional circulating CD4+ and CD8+ T cell responses in gastric cancer patients. a, IFN-y ELISPOT of patient 035 PBMCs stimulated with individualized peptides to assess the neoantigen-specific T cell responses. No peptide media and phytohemagglutinin (PHA) were used as negative control and positive control, respectively. b, ICS of PBMCs after neoantigens stimulation. PBMCs were pre-gated based on CD3+ and CD8+ T cells. Cytokine-producing neoantigen-specific $T$ cells expressed CD45RO, demonstrating an antigen-experienced memory T cell phenotype. $\mathrm{C}$, In vitro measurement of intracellular IFN- $\gamma+$, TNF- $a+$ and IL-2+ CD 4+ T cells in PBMCs from patient 035 who was treated with individualized peptides. $d$, In vitro measurement of intracellular IFN- $\gamma+$, TNF- $a+$ and IL-2+ CD8+ T cells in patient 035 PBMCs which was stimulated with individualized peptides. e, Median 
percentage of cytokine production across 21 patients by CD4+ T cells stimulated in vitro with immunized peptides. Data was analyzed by paired two-tailed Student's t-test. ${ }^{* \star} P<0.01$. f, Nanovaccines induced persistence of immune responses, as indicated by level of IFN-y in PBMCs at week 52 after nanovaccines therapy among 12 patients. The number of responsive peptides for each patient is represented as orange stacked column at week 52 and the blue stacked column represents the number of no-responsive peptides. $\mathrm{g}$, The PBMCs of patient 056 at different time points were restimulated with immunized peptides overnight. Then the IFN- $\gamma$ concentration in culture supernatant was measured by IFN- $\gamma$ CBA assay. h, ICS of IFN-y and TNF-a cytokine-producing CD4+ T cells in PBMCs from patient 056 after one round pre-stimulation at the specific time point. i, Pie charts show that total CD8+ T cell responses positive for 1 or 2 cytokines (IFN-y, TNF-a or IL-2) at week 18 and week 110 after vaccination initiation in patient 002. Bar graphs show the absolute frequencies of ASP pool-reactive CD8+ T cells producing 1 or 2 cytokines.

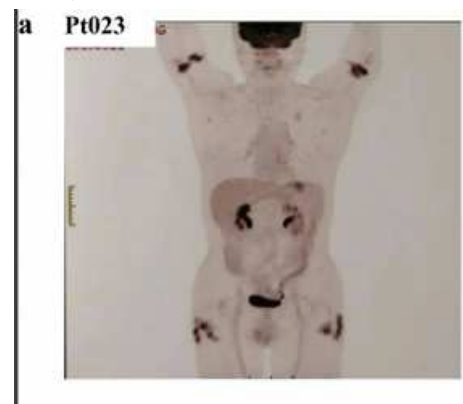

c Pt023

Post-Vac (Week 46)

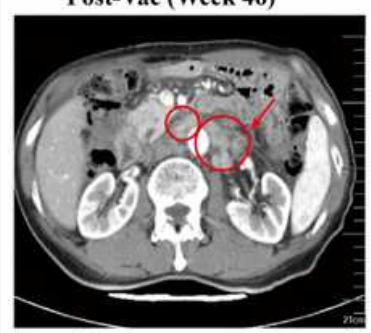

Post-Vac (Week 46)

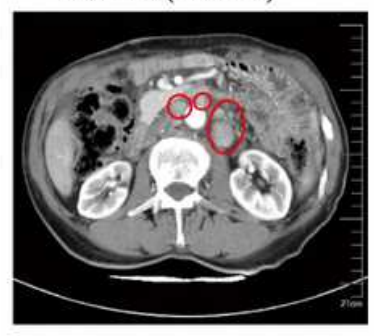

b Pt023

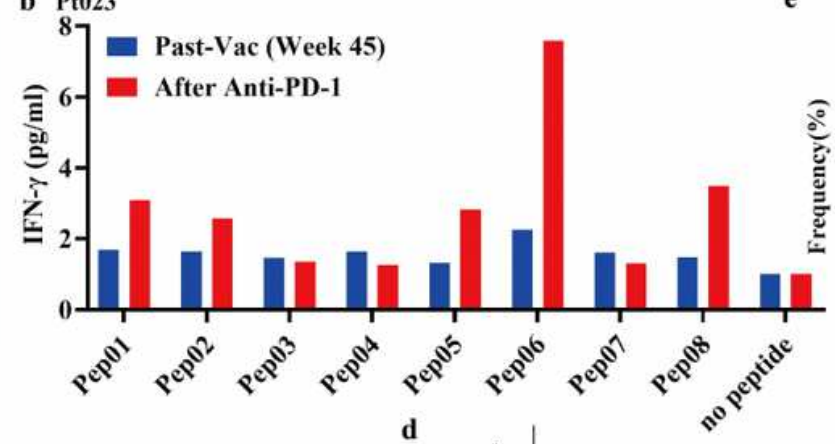

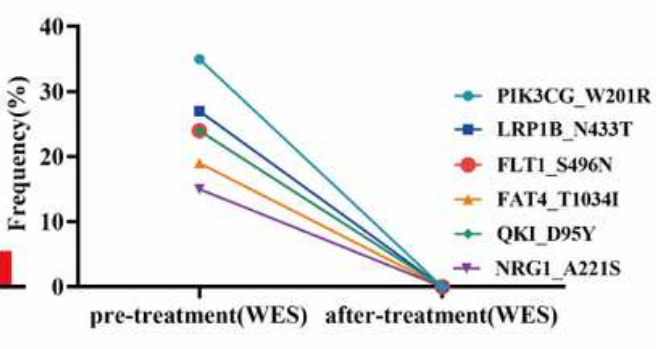

After Anti-PD-1

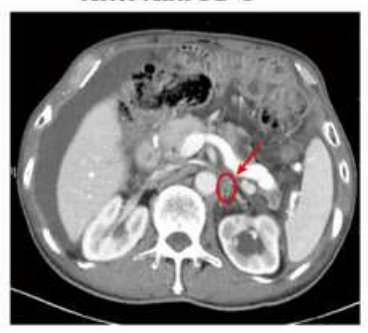

After Anti-PD-1

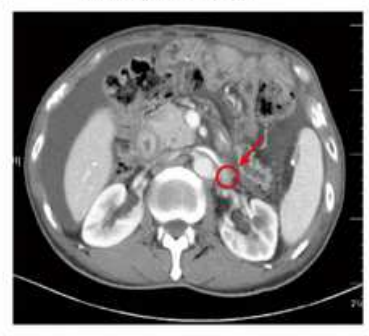

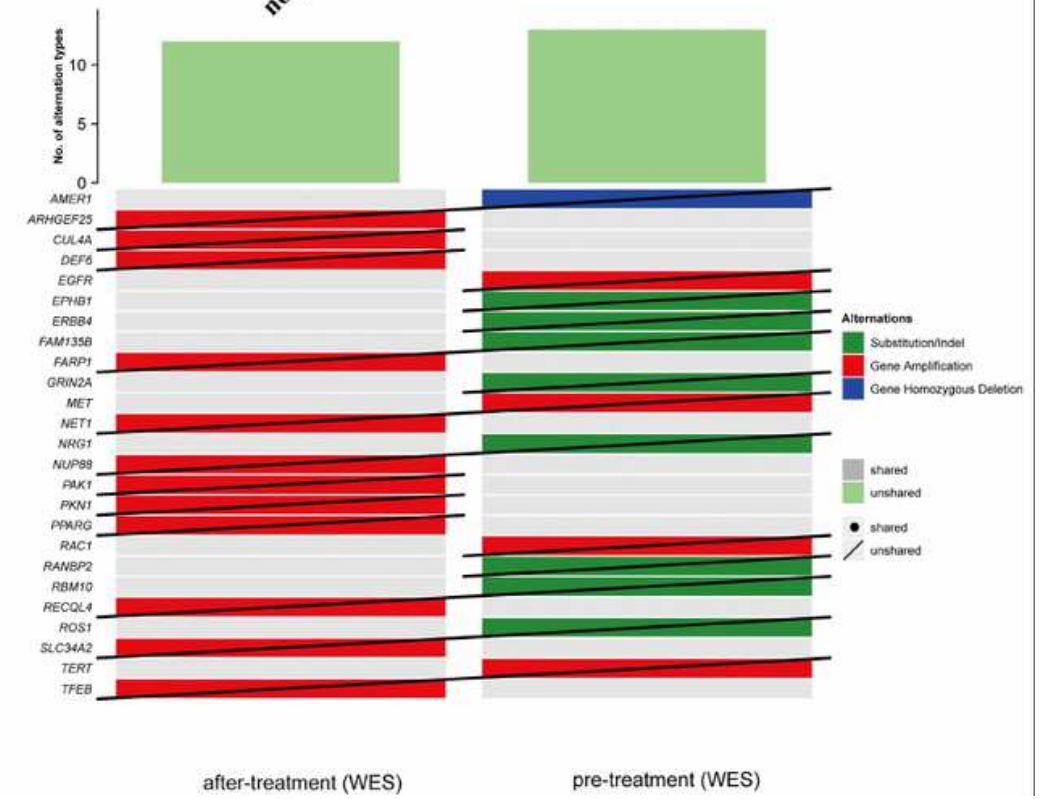

after-treatment (WES)

pre-treatment (WES)

\section{Figure 6}

Clinical and immune responses to anti-PD-1 blockade alone in patient 023 who relapsed after personalized PNVAC. a, In patient 023, PET/CT scan revealed inflammatory uptake at the vaccinated sites. $b$, The neoantigen-specific $T$ cell responses were enhanced after PD- 1 blockade in patient 023 who relapsed after vaccination. c, Computed tomography (CT) scans showed that after 3 doses of anti-PD-1 blockade immunotherapy, multiple metastatic lymph nodes shrank, but ascites appeared. $d$, Comparison 
of somatic mutations between primary tumor and relapsed lesion detected using WES. e, Comparison of gene mutations corresponding to immunized neoantigens between primary tumor and progressed lesion in patient 023.

\section{Supplementary Files}

This is a list of supplementary files associated with this preprint. Click to download.

- ExtendedFIGS1.pdf

- ExtendedFIGS2.pdf

- ExtendedFIGS3.pdf

- ExtendedFIGS4.pdf

- ExtendedFIGS5.pdf

- ExtendedFIGS6.pdf

- ExtendedFIGS7.pdf

- ExtendedFIGS8.pdf

- ExtendedFIGS9.pdf

- ExtendedFIGS10.pdf

- SupplementaryTable1Characteristicsofpatients.xlsx

- SupplementaryTable2Individualizedneoantigenforeachpatient.xlsx

- SupplementaryTable3AdverseEvents.xIsx

- SupplementaryTable4QC1.xlsx

- SupplementaryrelatedfileTheprotocolforclinicaltrial.docx 